\title{
Investigation of the double-lobed sources of the Cygnus constellation core
}

\author{
J. Saponara ${ }^{1}$, P. Benaglia ${ }^{1}$, I. Andruchow ${ }^{2,3}$, C. H. Ishwara-Chandra ${ }^{4}$, H. T. Intema ${ }^{5,6}$ \\ ${ }^{1}$ Instituto Argentino de Radioastronomía, CONICET-CICPBA-UNLP, CC5 (1897) Villa Elisa, Prov. de Buenos Aires, Argentina \\ ${ }^{2}$ Facultad de Cs. Astronómicas y Geofísicas, Universidad Nacional de La Plata \\ ${ }^{3}$ Instituto de Astrofísica de La Plata (UNLP - CONICET, CCT La Plata) \\ ${ }^{4}$ National Centre for Radio Astrophysics, Tata Institute of Fundamental Research, Pune University Campus, Pune, 411007, India \\ ${ }^{5}$ International Centre for Radio Astronomy Research, Curtin University, Bentley, WA 6102, Australia \\ ${ }^{6}$ Leiden Observatory, Leiden University, Niels Bohrweg 2, 2333 CA Leiden, the Netherlands
}

\begin{abstract}
We present a collection of double-lobed sources towards a 20 sq deg area of the Cygnus region at the northern sky, observed at 325 and $610 \mathrm{MHz}$ with the Giant Metrewave Radio Telescope. The 10" resolution achieved at $325 \mathrm{MHz}$ is 5.5 times better than previous studies, while at $610 \mathrm{MHz}$ these are the first results ever of such a large area, mapped with 6" angular resolution. After a thorough visual inspection of the images at the two bands, we found 43 double-lobed source candidates, proposed as such due to the presence of two bright peaks, within a few arcminutes apart, joined by a bridge or a central nucleus. All but two are presented here as a double-lobed candidates for the first time. Thirty-nine of the candidates were covered at both bands, and we provide the spectral index information for them. We have searched for positional coincidences between the detected sources/components and other objects from the literature, along the electromagnetic spectrum. Twenty-three candidates possess radio counterpart(s), 12 present infrared counterparts, and one showed an overlapping X-ray source. We analysed each candidate considering morphology, counterparts, and spectral indices. Out of the 43 candidates, 37 show characteristics compatible with an extragalactic nature, two of probably Galactic origin, three remain as dubious cases, though with feature(s) compatible with an extragalactic nature, and the remaining one, evidence of physically unrelated components. The median spectral index of the 40 putative extragalactic sources is -1.0 . Their celestial surface density at $610 \mathrm{MHz}$ resulted in 1.9 per sq deg, across a region lying at the Galactic plane.
\end{abstract}

Keywords: Catalogues - Radio continuum: general - galaxies: active - galaxies: field galaxies

\section{INTRODUCTION}

The Cygnus constellation covers a relevant area of the northern sky and harbours many stellar associations and clusters. The Cygnus OB2 association, at its centre, is a well studied rich region with several massive stars, as known since the early work of Münch \& Morgan (1953); Schulte (1956b,a, 1958); Reddish et al. (1966); Knödlseder et al. (2002); Albacete Colombo et al. (2007); see also the review of Reipurth $\&$ Schneider (2008). Recent radio results at arcsecond resolution were presented in Benaglia et al. (2020b), further including Cyg OB8 and OB9, using Giant Metrewave Radio Telescope (GMRT) data. Some massive stars towards these regions show significant radio emission, like the Wolf-Rayet stars WR 146, 147 for instance, with their colliding winds, that generate non-thermal (NT) radio fluxes (e.g., Williams et al., 1997; Benaglia et al., 2020a, and many references therein). Besides massive, early-type stars, other types of objects were studied in this region, like the proto-planetary disk-like sources (Isequilla et al., 2019) and young stellar objects (YSO, Isequilla et al., 2020). Some types of Galactic sources, related to stellar systems at different stages, present jet/outflows morphology. Interesting examples are those related to protostars, YSO and Herbig-Haro objects (HH, see Anglada et al., 2018, the latest review). In the case of HH80/81 and jets, besides NT spectral indices as in other cases, Carrasco-González et al. (2010) measured important linear polarisation, confirming synchrotron emission. We inspect the GMRT images used by Benaglia et al. (2020b) to build their catalogue. We identified several faint radio sources with double-lobed mophologies; many of them observed for the first time. And, even though they are towards the Galactic plane, some of them might have an extragalactic nature.

Regular members of the extragalactic zoo, the Active Galactic Nuclei (AGN) are notably strong sources with a spectral energy distribution (SED) ranging from gamma to 
radio wavelengths (Brown et al., 2019); generally the SED presents a peak at UV and significant luminosity contribution at the infrared and X-rays wavelengths. Radio galaxies are a subclass of AGNs. The radio continuum maps usually reveal a bright centre (core), jets and lobes whose extension can reach Mpc. The radio galaxies were classified into two main kinds, the Fanaroff-Riley I and II (FRI, FRII, Fanaroff \& Riley, 1974). The FRIs present their low brightness regions further from the core than their high brightness regions, while it is in the other way round for the FRIIs. At the FRII high brightness regions, the jets are decelerated by the interaction with the circumgalactic medium. Both classic examples are M84 and 3C175, respectively (e.g., Laing et al., 1983). But the classifications account for the standard type of radio galaxies. Many others with different morphologies, such us X-shapes (Leahy \& Williams, 1984), collimated jets, and also some of them with inner double-lobed radio structure as well as a larger outer double-lobed structure, called 'double-double' radio galaxies (DDRGs) (Schoenmakers et al., 2000; Brocksopp et al., 2011), were also reported. In addition, the majority of the studies were performed out of the Galactic plane. The optical identification is difficult against a region crowded with emitters at that spectral range, and the lack of high resolution and deep radio images are a problem as well.

An investigation of double-lobed radio sources in the Cygnus region was lacking. In this work, we present the study of the double-lobed type sources in the central part of the Cygnus region, near the Galactic plane, to characterise those with extragalactic features; many of them are reported for the first time in this work. GMRT observations are introduced in Section 2. Section 3 describes the identification processes, and Section 4 the search for counterparts. The discussion is presented in Section 5, and the main conclusions are listed in Section 6.

\section{RADIO OBSERVATIONS}

Along the past years, different radio surveys were focused on the Galactic plane and thus on the Cygnus area. Jones et al. (1988) using the Very Large Array (VLA) carried out continuum observations at $1.4 \mathrm{GHz}\left(b=0^{\circ}\right)$. The resolution achieved in this survey was up to $4^{\prime \prime}$ and the findings complete to about 30-mJy peak in flux density. The results were complemented by Zoonematkermani et al. (1990), for $|b|<0.8^{\circ}$, with similar angular resolution and flux limit. In 1996, with the Texas Interferometer at $365 \mathrm{MHz}$, Douglas et al. (1996a) imaged the area at arcminutes scale above flux densities of $0.25-0.4 \mathrm{Jy}$; and Taylor et al. (1996) carried out Westerbork Synthesis Radio Telescope (WSRT) observations at $327 \mathrm{MHz}$ for $|b|<1.6^{\circ}$ (WSRT Galactic Plane survey or WSRTGP), achieving an angular resolution of $\sim 1^{\prime}$ and detecting sources brighter than $10 \mathrm{mJy}^{\text {beam }}{ }^{-1}$. This same interferometer was used to observe the Cyg OB2 region and build the 325 and $1400 \mathrm{MHz}$ continuum survey, published by Setia Gunawan et al. (2003). The attained angular resolutions were $13^{\prime \prime}$ and $55^{\prime \prime}$ with $5 \sigma$ flux density limits of $\sim 2 \mathrm{mJy}$ and $10-15 \mathrm{mJy}$, respectively. Very recently, Morford et al. (2020) published results of a deep field mapping of the Cyg OB2 association at $21 \mathrm{~cm}$, attaining an angular resolution of 180 mas and rms as low as $21 \mu \mathrm{Jy}$.

In order to achieve both high-angular resolution and sensitivity below the mJy threshold, the central $19.7 \mathrm{sq}$ deg of the Cygnus constellation (see Fig. 1) were observed using two bands, centred at 325 and $610 \mathrm{MHz}$, with the Giant Metrewave Radio Telescope. The data were collected during 172 hours along 2013-2017, using a $32 \mathrm{MHz}$ bandwidth, and were calibrated and processed uniformly, using the SPAM routines (Intema, 2014). For full details on observations, calibration and imaging, see Benaglia et al. (2020b). The SPAM routines provided also the final mosaics, one at each band, for which a robust weighting of -1 was chosen. We have made use here of the images that are based on those observations. The mosaics present a mean rms of $0.5 \mathrm{mJy}^{\text {beam }}{ }^{-1}$ and 0.2 $\mathrm{mJy}$ beam $^{-1}$ at 325 and $610 \mathrm{MHz}$, but we note that the rms value locally varies depending on the presence of extended and/or diffuse emission, up to 0.9 and $0.5 \mathrm{mJy} \mathrm{beam}^{-1}$. The resultant synthesised beams at each frequency at full resolution were $10^{\prime \prime} \times 10^{\prime \prime}$, and $6^{\prime \prime} \times 6^{\prime \prime}$. The final mosaic image sizes resulted in $(6487 \times 6573)$, and $(12580 \times 13837)$ pixels, of sizes $2.5^{\prime \prime}$ and $1.5^{\prime \prime}$, respectively. We note that this paper is the fourth of a series derived from the analysis of the Cygnus region through GMRT observations, see Benaglia et al. (2020a,b, 2021). Besides the SPAM mosaics, we used, for some specific regions, complementary images built with the astronomical image processing system (AIPS, Greisen, 2003), following standard procedures, and different weightings (see Benaglia et al., 2021, for details).

\section{IDENTIFYING DOUBLE-LOBED SOURCES IN THE CYGNUS REGION}

For the present study, we visually searched in the image mosaics for objects with two bright peaks, within a few arcminutes apart, joined by a bridge or path of emission, either diffuse or intense, or with a central nucleus. We proposed them as 'double-lobed' candidates ${ }^{1}$. To take into consideration the diverse local rms, we made individual images of each source, showing simultaneously the continuum emission at both bands when possible, see Figs. A1, A2, A3, A4, A5 and A6.

The spectral index $(\alpha)$ of a source is a key parameter to reveal its nature. It is defined in terms of the integrated flux density $S$ at frequency $v$; here we used the convention $S_{v} \propto v^{\alpha}$.

\footnotetext{
${ }^{1}$ In Benaglia et al. (2020b), the authors grouped candidates for double sources (not necessarily bi-lobed), using a different approach to build the sample: the pairs of sources were selected from the Gaussian fits resultant of running a source finding routine, and the selection process constraints consisted of a limiting ratio of component integrated flux densities and a limiting separation $\left(<=2^{\prime}\right)$.
} 


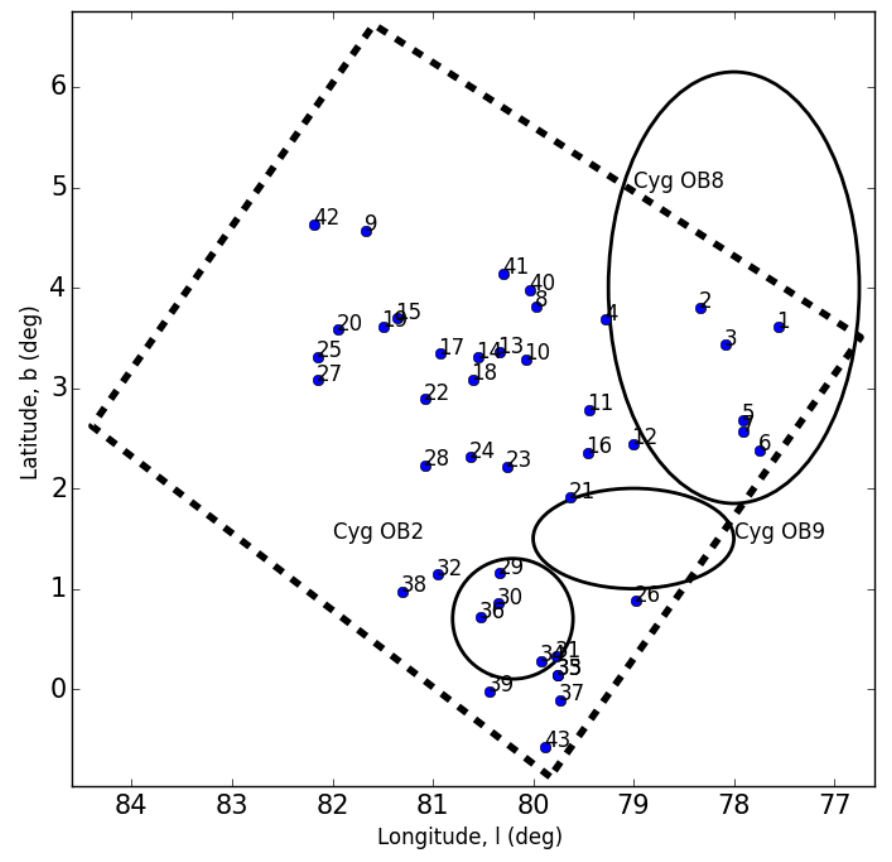

Figure 1. Observed area of the Cygnus constellation marked with a black dashed contour box, over the identified stellar associations Cygnus OB2 and Cygnus OB8 as well as Cygnus OB9. The blue dots are the double-lobed sources presented in this paper, with ID numbers as in Table 1.

To build the spectral index map of sources, we convolved and regridded the $610 \mathrm{MHz}$ image to exactly the same synthesised beam and grid as the $325 \mathrm{MHz}$ one (thus the pixel size in both maps is the same, $2.5^{\prime \prime} \times 2.5^{\prime \prime}$ ). The MIRIAD task CGCURS was used to estimate the integrated flux densities of the identified sources, within the region defined by 3 times the local rms. The main radio properties of the identified double-lobed sources are listed in Table 1, being

Column (1) - Source/candidate number (\#).

Column (2) - Source ID. The sources are listed in increasing order of right ascension: first those with GMRT data at both bands, and second the ones with only $610 \mathrm{MHz}$ data.

Column (3) - Label corresponding to the type of source centre, as (B): detected bridge; $(\mathrm{Nu})$ : detected nucleus candidate; $(\mathrm{G})$ measured geometric centre. The orientation of the lobes is quoted by means of cardinal celestial points (N, S, E, W, NW, SE, NE and SW).

Columns $(4,5)-\mathrm{J} 2000$ position in right ascension and declination of the components identified in Column 3.

Columns (6) - The total extension of the sources derived considering the distance between the extreme of the outermost contour $(=3 \mathrm{rms})$ of each lobe at $325 \mathrm{MHz}$. Estimated errors: \pm 2 arcsec.

Columns (7)-(9) - Integrated flux density of each component, at the $325 \mathrm{MHz}$ image, at the $610 \mathrm{MHz}$ image convolved to the $325 \mathrm{MHz}$ beam, and at the $610 \mathrm{MHz}$ full resolution image, respectively. The error corresponds to $3 \sigma$ (with $\sigma$, local rms). When a source was detected at one frequency only, the upper limit -at the other frequency band-corresponds to 3 times the local rms noise. Whenever was possible to calculate the integrated fluxdensity of the bridge emission, we measured its flux above $3 \sigma$, checking not to introduce the emission from the lobes. Since all nuclei sizes were encompassed by one beam, their integrated flux densities were calculated over the beam-size area.

Column (10) - Spectral index $\alpha$ of the component identified in Column 3.

Column (11) - Total spectral index $\alpha_{T}$ of the doublelobed source candidate; calculated using the flux sum of each source component. 
Table 1 Position, integrated flux density and spectral index of double-lobed sources components, and total spectral index. Nu: nucleus, G: geometric centre, B: bridge. $S C_{610 \mathrm{MHz}}$ refers to the integrated flux density obtained from the $610 \mathrm{MHz}$ image convolved to the $325 \mathrm{MHz}$ beam.

\begin{tabular}{|c|c|c|c|c|c|c|c|c|c|c|}
\hline \# & ID & & $\begin{array}{r}R A(\mathrm{~J} 2000) \\
(\mathrm{hms})\end{array}$ & $\begin{array}{r}\operatorname{Dec}(\mathrm{J} 2000) \\
(\mathrm{dms})\end{array}$ & $\begin{array}{c}\text { Total } \\
\text { size }\left({ }^{\prime \prime}\right)\end{array}$ & $\begin{array}{r}S_{325 \mathrm{MHz}} \\
(\mathrm{mJy})\end{array}$ & $\begin{array}{r}S C_{610 \mathrm{MHz}} \\
(\mathrm{mJy})\end{array}$ & $\begin{array}{r}S_{610 \mathrm{MHz}} \\
(\mathrm{mJy})\end{array}$ & $\begin{array}{l}\alpha_{610 \mathrm{MHz}}^{325 \mathrm{MHz}} \\
\end{array}$ & $\alpha_{T}$ \\
\hline \multirow[t]{3}{*}{$\# 1$} & J201255+404423 & B & $20: 12: 55.00$ & $40: 44: 23.0$ & 60 & $4.7 \pm 1$ & $3.5 \pm 1.5$ & $1.8 \pm 0.4$ & & \\
\hline & & $\mathrm{N}$ & :55.44 & :33.3 & & $36 \pm 4$ & $22 \pm 4$ & $24 \pm 4$ & $-0.8 \pm 0.3$ & $-0.8 \pm 0.4$ \\
\hline & & S & :54.88 & :01.5 & & $72 \pm 12$ & $42 \pm 7$ & $47 \pm 4$ & $-0.8 \pm 0.4$ & \\
\hline \multirow[t]{3}{*}{$\# 2$} & J201418+412931 & $\mathrm{B}$ ? & $20: 14: 18.40$ & $41: 29: 31.0$ & 45 & $5.4 \pm 2$ & $<0.3$ & $<0.8$ & & \\
\hline & & $\mathrm{NE}$ & $: 19.13$ & $: 37.6$ & & $51 \pm 1$ & $27 \pm 5$ & $30 \pm 4$ & $-1.1 \pm 0.3$ & $-1.2 \pm 0.3$ \\
\hline & & SW & $: 17.43$ & :23.5 & & $77 \pm 1$ & $35 \pm 7$ & $39 \pm 5$ & $-1.2 \pm 0.3$ & \\
\hline \multirow[t]{3}{*}{$\# 3$} & $\mathrm{~J} 201512+410457$ & G & 20:15:11.20 & 41:04:57.0 & 55 & $<0.2$ & $<0.7$ & $<0.08$ & & \\
\hline & & $\mathrm{N}$ & $: 11.39$ & $: 05: 10.2$ & & $29 \pm 6$ & $17 \pm 2$ & $18 \pm 2$ & $-0.8 \pm 0.4$ & $-0.9 \pm 0.4$ \\
\hline & & S & :11.07 & $: 04: 38.5$ & & $14 \pm 2$ & $7 \pm 1$ & $8 \pm 1$ & $-1.1 \pm 0.3$ & \\
\hline \multirow[t]{3}{*}{$\# 4$} & $\mathrm{~J} 201739+421243$ & $\mathrm{Nu}$ & $20: 17: 39.24$ & $42: 12: 43.3$ & 66 & $2 \pm 0.5$ & $3 \pm 1$ & $3 \pm 1$ & & \\
\hline & & $\mathrm{N}$ & $: 40.00$ & $: 13: 12.0$ & & $20 \pm 2$ & $5 \pm 2$ & $6 \pm 1$ & $-2.2 \pm 0.6$ & $-2.0 \pm 0.4$ \\
\hline & & S & :38.60 & $: 12: 26.6$ & & $36 \pm 4$ & $8 \pm 1$ & $9 \pm 1$ & $-2.4 \pm 0.3$ & \\
\hline \multirow[t]{3}{*}{$\# 5$} & $\mathrm{~J} 201758+403100$ & $\mathrm{Nu}$ & $20: 17: 58.00$ & 40:31:00.0 & 72 & $35 \pm 4$ & $14 \pm 2$ & $15 \pm 1$ & & \\
\hline & & NE & $: 18: 00.16$ & $: 31: 07.8$ & & $278 \pm 15$ & $131 \pm 4$ & $138 \pm 4$ & $-1.2 \pm 0.1$ & $-1.3 \pm 0.1$ \\
\hline & & SW & $: 17: 54.96$ & $: 30: 33.5$ & & $217 \pm 11$ & $84 \pm 3$ & $93 \pm 3$ & $-1.5 \pm 0.1$ & \\
\hline \multirow[t]{3}{*}{$\# 6$} & J201829+402714 & B & 20:18:28.61 & $40: 27: 14.0$ & 25 & $2 \pm 1$ & $7 \pm 3$ & $3 \pm 1$ & & \\
\hline & & $\mathrm{N}$ & :28.46 & :20.5 & & $48 \pm 8$ & $24 \pm 6$ & $20 \pm 1$ & $-1.1 \pm 0.5$ & $-0.9 \pm 0.5$ \\
\hline & & S & :28.62 & :05.6 & & $24 \pm 4$ & $10 \pm 2$ & $8 \pm 1$ & $-1.4 \pm 0.4$ & \\
\hline \multirow[t]{3}{*}{$\# 7$} & $\mathrm{~J} 201847+401230$ & B & $20: 18: 46.64$ & $40: 12: 30.0$ & 45 & $15 \pm 5$ & $4 \pm 1$ & $3 \pm 1$ & & \\
\hline & & $\mathrm{N}$ & $: 46.76$ & :38.9 & & $100 \pm 20$ & $25 \pm 4$ & $24 \pm 3$ & $-2.2 \pm 0.4$ & $-2.2 \pm 0.4$ \\
\hline & & S & $: 47.05$ & :19.4 & & $45 \pm 10$ & $10 \pm 2$ & $9 \pm 1$ & $-2.4 \pm 0.5$ & \\
\hline \multirow[t]{3}{*}{ \#8 } & J201910+425136 & $\mathrm{Nu}$ & 20:19:09.33 & $42: 51: 36.25$ & 120 & $<0.6$ & $<0.03$ & $0.8 \pm 0.3$ & & \\
\hline & & $\mathrm{N}$ & :08.01 & $: 52: 28.4$ & & $9 \pm 3$ & $3 \pm 1$ & $4 \pm 1$ & $-1.7 \pm 0.7$ & $-1.3 \pm 0.4$ \\
\hline & & S & $: 10.25$ & $: 50: 37.9$ & & $180 \pm 30$ & $81 \pm 18$ & $84 \pm 12$ & $-1.3 \pm 0.4$ & \\
\hline \multirow[t]{3}{*}{$\# 9$} & $\mathrm{~J} 202058+444050$ & G & $20: 20: 58.00$ & $44: 40: 50.0$ & 120 & $<1$ & $<0.01$ & $<0.15$ & & \\
\hline & & $\mathrm{N}$ & $: 57.65$ & $: 41: 30.5$ & & $610 \pm 35$ & $404 \pm 16$ & $403 \pm 48$ & $-0.6 \pm 0.1$ & $-0.6 \pm 0.1$ \\
\hline & & $S$ & :57.90 & $: 40: 05.0$ & & $20 \pm 1$ & $19 \pm 1$ & $22 \pm 2$ & $-0.1 \pm 0.1$ & \\
\hline \multirow[t]{3}{*}{ \#10 } & $\mathrm{J} 202151+423847$ & G & $20: 21: 51.00$ & $42: 38: 47.0$ & 60 & $0.3 \pm 0.1$ & $1.6 \pm 0.2$ & $1.3 \pm 0.2$ & & \\
\hline & & $\mathrm{E}$ & $: 54.20$ & :32.8 & & $28 \pm 3$ & $15 \pm 1$ & $19 \pm 1$ & $-0.9 \pm 0.2$ & $-0.9 \pm 0.2$ \\
\hline & & W & $: 48.30$ & :57.6 & & $16 \pm 1$ & $8 \pm 1$ & $8 \pm 0.4$ & $-1.1 \pm 0.2$ & \\
\hline \multirow[t]{3}{*}{ \#11 } & J202209+415033 & $\mathrm{Nu}$ & $20: 22: 09.00$ & 41:50:33.0 & 60 & $0.7 \pm 0.4$ & $1 \pm 0.4$ & $0.7 \pm 0.1$ & & \\
\hline & & $\mathrm{N}$ & $: 09.30$ & $: 47.8$ & & $9 \pm 1$ & $6 \pm 1$ & $5 \pm 1$ & $-0.6 \pm 0.4$ & $-0.6 \pm 0.4$ \\
\hline & & S & :09.60 & :10.3 & & $9.5 \pm 1$ & $6 \pm 1$ & $5 \pm 1$ & $-0.7 \pm 0.3$ & \\
\hline \multirow[t]{3}{*}{$\# 12$} & $\mathrm{~J} 202218+411728$ & B & $20: 22: 18.00$ & 41:17:28.0 & 60 & $2 \pm 1$ & $3 \pm 1$ & $2 \pm 0.4$ & & \\
\hline & & NW & $: 17.00$ & :40.7 & & $16 \pm 2$ & $9 \pm 1$ & $9 \pm 1$ & $-0.9 \pm 0.3$ & $-0.7 \pm 0.3$ \\
\hline & & SE & $: 19.28$ & $: 12.4$ & & $10 \pm 1$ & $6 \pm 0.5$ & $5 \pm 0.5$ & $-0.8 \pm 0.2$ & \\
\hline
\end{tabular}


Table 1 continued.

\begin{tabular}{|c|c|c|c|c|c|c|c|c|c|c|}
\hline 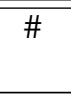 & $\overline{\mathrm{ID}}$ & & $\begin{array}{r}R A(\mathrm{~J} 2000) \\
(\mathrm{hms}) \\
\end{array}$ & $\begin{array}{r}\operatorname{Dec}(\mathrm{J} 2000) \\
(\mathrm{dms})\end{array}$ & $\begin{array}{c}\text { Total } \\
\text { size }\left({ }^{\prime \prime}\right)\end{array}$ & $\begin{array}{r}S_{325 \mathrm{MHz}} \\
(\mathrm{mJy})\end{array}$ & $\begin{array}{r}S C_{610 \mathrm{MHz}} \\
(\mathrm{mJy})\end{array}$ & $\begin{array}{r}S_{610 \mathrm{MHz}} \\
(\mathrm{mJy})\end{array}$ & $\begin{array}{c}\alpha_{610 \mathrm{MHz}}^{325 \mathrm{MHz}} \\
\end{array}$ & $\alpha_{T}$ \\
\hline \multirow[t]{3}{*}{$\# 13$} & J202222+425409 & B & $20: 22: 22.00$ & $42: 54: 09.0$ & 72 & $5 \pm 3$ & $4.6 \pm 1$ & $2.6 \pm 0.4$ & & \\
\hline & & $\mathrm{E}$ & :24.06 & $: 07.6$ & & $191 \pm 33$ & $106 \pm 17$ & $106 \pm 10$ & $-0.9 \pm 0.4$ & $-0.9 \pm 0.4$ \\
\hline & & W & $: 19.14$ & $: 10.1$ & & $329 \pm 54$ & $179 \pm 26$ & $180 \pm 17$ & $-1.0 \pm 0.3$ & \\
\hline \multirow[t]{3}{*}{$\# 14$} & $\mathrm{~J} 202315+430252$ & B & $20: 23: 15.00$ & $43: 02: 52.0$ & 45 & $4 \pm 2$ & $1.7 \pm 1$ & $1 \pm 0.4$ & & \\
\hline & & $\mathrm{NE}$ & $: 14.79$ & :03:03.0 & & $20 \pm 4$ & $12 \pm 2$ & $12 \pm 1$ & $-0.8 \pm 0.4$ & $-1.0 \pm 0.4$ \\
\hline & & SW & $: 16.17$ & $: 02: 42.1$ & & $20 \pm 3$ & $10 \pm 1$ & $10 \pm 1$ & $-1.1 \pm 0.3$ & \\
\hline \multirow[t]{3}{*}{$\# 15$} & $\mathrm{~J} 202359+435525$ & $\mathrm{Nu}$ & $20: 23: 58.61$ & $43: 55: 25.0$ & 72 & $1.7 \pm 0.3$ & $2 \pm 0.3$ & $2 \pm 0.2$ & & \\
\hline & & $\mathrm{NE}$ & $24: 02.38$ & $: 52.8$ & & $40 \pm 3$ & $21 \pm 2$ & $22 \pm 1$ & $-1.0 \pm 0.2$ & $-1.0 \pm 0.2$ \\
\hline & & SW & $23: 55.87$ & :03.0 & & $46 \pm 4$ & $23 \pm 2$ & $24 \pm 1$ & $-1.1 \pm 0.2$ & \\
\hline \multirow[t]{3}{*}{ \#16 } & $\mathrm{J} 202403+413611$ & $\mathrm{Nu}$ & $20: 24: 03.00$ & 41:36:11.0 & 90 & $20 \pm 2$ & $12 \pm 2$ & $11 \pm 2$ & & \\
\hline & & NW & $: 01.05$ & $: 36: 43.1$ & & $35 \pm 3$ & $22 \pm 1$ & $25 \pm 1$ & $-0.7 \pm 0.1$ & $-0.7 \pm 0.2$ \\
\hline & & SE & $: 04.15$ & $: 35: 37.2$ & & $37 \pm 2$ & $25 \pm 1$ & $28 \pm 1$ & $-0.6 \pm 0.1$ & \\
\hline \multirow[t]{3}{*}{ \#17 } & $\mathrm{J} 202413+432220$ & B & $20: 24: 13.00$ & $43: 22: 20.0$ & 72 & $65 \pm 13$ & $19 \pm 3$ & $22 \pm 2$ & & \\
\hline & & NE & $: 15.84$ & $: 33.5$ & & $488 \pm 70$ & $258 \pm 25$ & $243 \pm 23$ & $-1.0 \pm 0.3$ & $-0.8 \pm 0.3$ \\
\hline & & SW & $: 10.76$ & $: 04.8$ & & $1164 \pm 212$ & $729 \pm 69$ & $724 \pm 56$ & $-0.7 \pm 0.3$ & \\
\hline \multirow[t]{3}{*}{$\# 18$} & $\mathrm{~J} 202422+425722$ & $\mathrm{~B}$ & $20: 24: 22.00$ & 42:57:22.0 & 50 & $20 \pm 3$ & $12 \pm 4$ & $8 \pm 2$ & & \\
\hline & & W & :20.86 & $: 27.7$ & & $176 \pm 26$ & $99 \pm 4$ & $95 \pm 9$ & $-0.9 \pm 0.2$ & $-1.0 \pm 0.3$ \\
\hline & & $\mathrm{E}$ & $: 23.48$ & $: 18.5$ & & $90 \pm 20$ & $44 \pm 8$ & $43 \pm 5$ & $-1.1 \pm 0.4$ & \\
\hline \multirow[t]{3}{*}{ \#19 } & $\mathrm{J} 202448+435956$ & B & $20: 24: 47.88$ & $43: 59: 56.0$ & 45 & $1.4 \pm 0.7$ & $0.7 \pm 0.3$ & $0.6 \pm 0.2$ & & \\
\hline & & $\mathrm{E}$ & :49.68 & $: 59: 50.0$ & & $47 \pm 6$ & $29 \pm 2$ & $29 \pm 2$ & $-0.8 \pm 0.2$ & $-0.8 \pm 0.2$ \\
\hline & & $\mathrm{W}$ & $: 46.76$ & $: 00: 00.4$ & & $15 \pm 1$ & $9 \pm 1$ & $9 \pm 1$ & $-0.8 \pm 0.2$ & \\
\hline \multirow[t]{3}{*}{$\# 20$} & $\mathrm{~J} 202625+442048$ & B & $20: 26: 25.00$ & $44: 20: 48.0$ & 60 & $2.7 \pm 0.7$ & $2 \pm 1$ & $1.4 \pm 0.3$ & & \\
\hline & & $\mathrm{NE}$ & $: 25.93$ & $: 21: 02.0$ & & $46 \pm 10$ & $25 \pm 4$ & $25 \pm 4$ & $-0.9 \pm 0.4$ & $-1.0 \pm 0.4$ \\
\hline & & SW & $: 23.83$ & :20:39.6 & & $34 \pm 6$ & $17 \pm 2$ & $18 \pm 2$ & $-1.1 \pm 0.3$ & \\
\hline \multirow[t]{3}{*}{ \#21 } & $\mathrm{J} 202632+412942$ & G & $20: 26: 32.62$ & $41: 29: 42.6$ & 45 & $6 \pm 1$ & $0.8 \pm 0.2$ & $0.8 \pm 1$ & & \\
\hline & & $\mathrm{E}$ & :34.48 & :47.0 & & $114 \pm 25$ & $54 \pm 10$ & $55 \pm 7$ & $-1.2 \pm 0.4$ & $-1.4 \pm 0.4$ \\
\hline & & W & :30.21 & $: 41.2$ & & $107 \pm 20$ & $39 \pm 7$ & $41 \pm 5$ & $-1.6 \pm 0.4$ & \\
\hline \multirow[t]{3}{*}{ \#22 } & $202645+431435$ & B & $20: 26: 45.00$ & $43: 14: 35.0$ & 138 & $<0.2$ & $<0.5$ & $<0.5$ & & \\
\hline & & $\mathrm{N}$ & $: 47.18$ & $: 16: 09.4$ & & $8 \pm 1$ & $2 \pm 0.3$ & $2 \pm 0.3$ & $-2.2 \pm 0.3$ & $-1.6 \pm 0.1$ \\
\hline & & $\mathrm{S}$ & $: 43.84$ & $: 14: 05.5$ & & $20 \pm 1$ & $8 \pm 0.3$ & $8 \pm 0.2$ & $-1.4 \pm 0.1$ & \\
\hline \multirow[t]{3}{*}{ \#23 } & $\mathrm{J} 202710+421113$ & G & $20: 27: 10.00$ & $42: 11: 13.0$ & 55 & $1 \pm 0.5$ & $0.8 \pm 0.2$ & $0.8 \pm 0.1$ & & \\
\hline & & $\mathrm{NE}$ & $: 11.36$ & :26.1 & & $59 \pm 9$ & $25 \pm 4$ & $23 \pm 3$ & $-1.4 \pm 0.3$ & $-1.3 \pm 0.3$ \\
\hline & & SW & $: 08.25$ & :09.8 & & $18 \pm 4$ & $8.7 \pm 0.2$ & $8.7 \pm 0.1$ & $-1.1 \pm 0.3$ & \\
\hline \multirow[t]{3}{*}{ \#24 } & $\mathrm{J} 202755+423215$ & G & $20: 27: 55.00$ & $42: 32: 15.0$ & 60 & $11 \pm 5$ & $0.9 \pm 0.5$ & $0.4 \pm 0.1$ & & \\
\hline & & $\mathrm{NE}$ & $: 57.12$ & $: 32: 30.6$ & & $349 \pm 56$ & $146 \pm 14$ & $337 \pm 30$ & $-1.4 \pm 0.3$ & $-1.4 \pm 0.2$ \\
\hline & & SW & $: 53.30$ & $: 31: 56.4$ & & $800 \pm 14$ & $337 \pm 32$ & $146 \pm 12$ & $-1.4 \pm 0.1$ & \\
\hline
\end{tabular}


Table 1 continued.

\begin{tabular}{|c|c|c|c|c|c|c|c|c|c|c|}
\hline \# & ID & & $\begin{array}{r}R A(\mathrm{~J} 2000) \\
(\mathrm{hms}) \\
\end{array}$ & $\begin{array}{r}\operatorname{Dec}(\mathrm{J} 2000) \\
(\mathrm{dms})\end{array}$ & $\begin{array}{c}\text { Total } \\
\text { size }\left({ }^{\prime \prime}\right)\end{array}$ & $\begin{array}{r}S_{325 \mathrm{MHz}} \\
(\mathrm{mJy})\end{array}$ & $\begin{array}{r}S C_{610 \mathrm{MHz}} \\
(\mathrm{mJy})\end{array}$ & $\begin{array}{r}S_{610 \mathrm{MHz}} \\
(\mathrm{mJy})\end{array}$ & $\alpha_{610 \mathrm{MHz}}^{325 \mathrm{MHz}}$ & $\alpha_{T}$ \\
\hline \multirow[t]{3}{*}{$\# 25$} & J202817+442114 & $\mathrm{B}$ & $20: 28: 17.00$ & $44: 21: 14.0$ & 60 & $1 \pm 0.3$ & $0.5 \pm 0.2$ & $0.7 \pm 0.3$ & & \\
\hline & & $\mathrm{NE}$ & $: 18.54$ & $: 21: 32.9$ & & $19 \pm 2$ & $10 \pm 1$ & $11 \pm 1$ & $-1.0 \pm 0.2$ & $-0.8 \pm 0.2$ \\
\hline & & SW & $: 16.40$ & $: 20: 48.0$ & & $25 \pm 2$ & $16 \pm 1$ & $15 \pm 1$ & $-0.7 \pm 0.2$ & \\
\hline \multirow[t]{3}{*}{ \#26 } & $\mathrm{J} 202859+402141$ & B & $20: 28: 59.00$ & $40: 21: 41.0$ & 60 & $<0.4$ & $1.6 \pm 1$ & $2.4 \pm 0.4$ & & \\
\hline & & $\mathrm{N}$ & :58.92 & $: 22: 05.5$ & & $18 \pm 4$ & $16 \pm 8$ & $15 \pm 1$ & $-0.2 \pm 0.8$ & $+0.4 \pm 0.7$ \\
\hline & & S & :59.00 & $: 21: 24.9$ & & $4 \pm 1$ & $10 \pm 1$ & $9.6 \pm 1$ & $+1.4 \pm 0.4$ & \\
\hline \multirow[t]{3}{*}{$\# 27$} & J202919+441341 & $\mathrm{G}$ & $20: 29: 18.00$ & $44: 13: 41.8$ & 60 & $<0.3$ & $<0.3$ & $<0.1$ & & \\
\hline & & NW & $: 14.97$ & $: 13: 39.7$ & & $145 \pm 14$ & $84 \pm 5$ & $85 \pm 4$ & $-0.9 \pm 0.2$ & $-0.9 \pm 0.2$ \\
\hline & & SE & :21.28 & $: 12: 21.4$ & & $10 \pm 1$ & $5 \pm 0.5$ & $5 \pm 0.5$ & $-1.1 \pm 0.2$ & \\
\hline \multirow[t]{3}{*}{ \#28 } & $\mathrm{J} 202943+425122$ & B & $20: 29: 43.00$ & $42: 51: 22.0$ & 40 & $2 \pm 1$ & $1 \pm 0.3$ & $1 \pm 0.3$ & & \\
\hline & & $\mathrm{E}$ & $: 44.84$ & :29.4 & & $17 \pm 3$ & $9 \pm 1$ & $9 \pm 1$ & $-1.0 \pm 0.3$ & $-0.9 \pm 0.4$ \\
\hline & & W & $: 42.24$ & :20.7 & & $23 \pm 4$ & $14 \pm 2$ & $13 \pm 1$ & $-0.8 \pm 0.3$ & \\
\hline \multirow[t]{3}{*}{ \#29 } & $\mathrm{J} 203201+413730$ & $\mathrm{G}$ & 20:32:01.00 & $41: 37: 30.0$ & 50 & $10 \pm 3$ & $4 \pm 1$ & $4 \pm 1$ & & \\
\hline & & $\mathrm{N}$ & :1.92 & :53.8 & & $159 \pm 10$ & $77 \pm 2$ & $73 \pm 2$ & $-1.1 \pm 0.1$ & $-1.2 \pm 0.1$ \\
\hline & & $\mathrm{S}$ & $: 0.91$ & $: 07.6$ & & $172 \pm 10$ & $76 \pm 2$ & $80 \pm 2$ & $-1.3 \pm 0.1$ & \\
\hline \multirow[t]{3}{*}{$\# 30$} & $\mathrm{~J} 203323+412720$ & B & $20: 33: 23.00$ & $41: 27: 20.0$ & 60 & $14 \pm 6$ & $8 \pm 3$ & $7 \pm 1$ & & \\
\hline & & W & $: 22.43$ & :25.3 & & $126 \pm 9$ & $56 \pm 3$ & $66 \pm 7$ & $-1.3 \pm 0.1$ & $-1.1 \pm 0.2$ \\
\hline & & $\mathrm{E}$ & :24.42 & $: 17.3$ & & $130 \pm 16$ & $67 \pm 6$ & $57 \pm 3$ & $-1.0 \pm 0.2$ & \\
\hline \multirow[t]{3}{*}{ \#31 } & $\mathrm{J} 203345+404015$ & $\mathrm{Nu}$ & $20: 33: 45.58$ & $40: 40: 15.7$ & 120 & $5 \pm 1$ & $3 \pm 1$ & $3 \pm 1$ & & \\
\hline & & $\mathrm{N}$ & :47.39 & $: 41: 00.2$ & & $111 \pm 8$ & $46 \pm 2$ & $48 \pm 2$ & $-1.4 \pm 0.1$ & $-1.3 \pm 0.2$ \\
\hline & & $\mathrm{S}$ & :43.63 & $: 39: 28.2$ & & $156 \pm 12$ & $71 \pm 4$ & $73 \pm 4$ & $-1.2 \pm 0.1$ & \\
\hline \multirow[t]{3}{*}{ \#32 } & $\mathrm{J} 203402+420615$ & $\mathrm{Nu}$ & $20: 34: 02.60$ & $42: 06: 15.4$ & 72 & $11 \pm 2$ & $5 \pm 1$ & $9 \pm 1$ & & \\
\hline & & $\mathrm{NE}$ & :04.09 & $: 06: 31.5$ & & $134 \pm 4$ & $60 \pm 1$ & $48 \pm 2$ & $-1.3 \pm 0.1$ & $-1.2 \pm 0.1$ \\
\hline & & SW & $: 01.31$ & $: 05: 59.2$ & & $86 \pm 7$ & $42 \pm 2$ & $73 \pm 4$ & $-1.1 \pm 0.1$ & \\
\hline \multirow[t]{3}{*}{ \#33 } & $\mathrm{J} 203413+403349$ & B & $20: 34: 12.97$ & $40: 33: 49.6$ & 60 & $3 \pm 1$ & $0.6 \pm 0.1$ & $0.6 \pm 0.2$ & & \\
\hline & & $\mathrm{N}$ & $: 13.28$ & $: 34: 13.2$ & & $25 \pm 4$ & $9 \pm 1$ & $9 \pm 2$ & $-1.6 \pm 0.3$ & $-1.5 \pm 0.3$ \\
\hline & & $\mathrm{S}$ & $: 12.28$ & $: 33: 34.8$ & & $51 \pm 8$ & $21 \pm 3$ & $21 \pm 4$ & $-1.4 \pm 0.3$ & \\
\hline \multirow[t]{3}{*}{ \#34 } & $\mathrm{J} 203425+404605$ & $\mathrm{Nu}$ & $20: 34: 25.80$ & $40: 46: 05.9$ & 60 & $39 \pm 5$ & $22 \pm 4$ & $22 \pm 3$ & & \\
\hline & & $\mathrm{W}$ & :23.00 & $: 45: 56.0$ & & $70 \pm 3$ & $35 \pm 2$ & $43 \pm 1$ & $-1.1 \pm 0.1$ & $-1.2 \pm 0.2$ \\
\hline & & $\mathrm{E}$ & :27.00 & $: 46: 02.0$ & & $54 \pm 2$ & $21 \pm 5$ & $28 \pm 1$ & $-1.5 \pm 0.4$ & \\
\hline \multirow[t]{3}{*}{ \#35 } & $\mathrm{J} 203431+413037$ & B & $20: 34: 30.84$ & $41: 30: 37.8$ & 60 & $3 \pm 1$ & $3 \pm 1$ & $1.5 \pm 0.2$ & & \\
\hline & & NW & :30.47 & :51.4 & & $33 \pm 3$ & $27 \pm 3$ & $29 \pm 2$ & $-0.3 \pm 0.3$ & $-0.4 \pm 0.3$ \\
\hline & & SE & $: 31.21$ & :25.6 & & $28 \pm 2$ & $18 \pm 1$ & $28 \pm 1$ & $+0.7 \pm 0.1$ & \\
\hline \multirow[t]{3}{*}{ \#36 } & $\mathrm{J} 203431+403332$ & B & $20: 34: 30.98$ & $40: 33: 32.1$ & 78 & $6 \pm 1$ & $1 \pm 0.1$ & $1 \pm 0.1$ & & \\
\hline & & $\mathrm{N}$ & :31.08 & $: 34: 14.2$ & & $55 \pm 4$ & $23 \pm 1$ & $22 \pm 1$ & $-1.4 \pm 0.1$ & $-1.2 \pm 0.1$ \\
\hline & & $\mathrm{S}$ & :32.04 & $: 32: 34.8$ & & $52 \pm 4$ & $29 \pm 1$ & $25 \pm 1$ & $-0.9 \pm 0.1$ & \\
\hline
\end{tabular}


Table 1 continued.

\begin{tabular}{|c|c|c|c|c|c|c|c|c|c|c|}
\hline 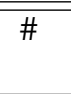 & $\overline{\mathrm{ID}}$ & & $\begin{array}{r}R A(\mathrm{~J} 2000) \\
(\mathrm{hms})\end{array}$ & $\begin{array}{r}\operatorname{Dec}(\mathrm{J} 2000) \\
(\mathrm{dms})\end{array}$ & $\begin{array}{c}\text { Total } \\
\text { size }\left({ }^{\prime \prime}\right)\end{array}$ & $\begin{array}{r}S_{325 \mathrm{MHz}} \\
(\mathrm{mJy})\end{array}$ & $\begin{array}{r}S C_{610 \mathrm{MHz}} \\
(\mathrm{mJy})\end{array}$ & $\begin{array}{r}S_{610 \mathrm{MHz}} \\
(\mathrm{mJy})\end{array}$ & $\overline{\alpha_{610 \mathrm{MHz}}^{325 \mathrm{MHz}}}$ & $\alpha_{T}$ \\
\hline \multirow[t]{3}{*}{$\# 37$} & $\mathrm{~J} 203529+403201$ & B & $20: 35: 29.78$ & $40: 32: 01.2$ & 70 & $4 \pm 0.4$ & $11 \pm 2$ & $5 \pm 1$ & & \\
\hline & & $\mathrm{NE}$ & $: 30.58$ & 40:32:06.9 & & $75 \pm 10$ & $35 \pm 4$ & $35 \pm 2$ & $-1.2 \pm 0.3$ & $-0.7 \pm 0.3$ \\
\hline & & SW & :29.11 & $40: 31: 59.9$ & & $49 \pm 10$ & $39 \pm 4$ & $12 \pm 1$ & $-0.4 \pm 0.4$ & \\
\hline \multirow[t]{3}{*}{ \#38 } & J203556+421744 & B & $20: 35: 57.11$ & $42: 17: 44.5$ & 78 & $122 \pm 32$ & $78 \pm 18$ & $101 \pm 8$ & & \\
\hline & & NW & :55.00 & $: 18: 06.2$ & & $3636 \pm 385$ & $1556 \pm 212$ & $1526 \pm 158$ & $-1.3 \pm 0.3$ & $-1.3 \pm 0.3$ \\
\hline & & SE & :58.80 & $: 17: 22.9$ & & $2863 \pm 227$ & $1334 \pm 167$ & $1318 \pm 104$ & $-1.2 \pm 0.2$ & \\
\hline \multirow[t]{3}{*}{ \#39 } & J203720+405941 & $\mathrm{Nu}$ & $20: 37: 20.73$ & $40: 59: 41.7$ & 60 & $2 \pm 1$ & $4 \pm 1$ & $2 \pm 1$ & & \\
\hline & & $\mathrm{N}$ & :22.00 & 41:00:08.3 & & $90 \pm 10$ & $43 \pm 4$ & $45 \pm 4$ & $-1.1 \pm 0.2$ & $-1.1 \pm 0.2$ \\
\hline & & $\mathrm{S}$ & $: 19.72$ & 40:59:15.1 & & $105 \pm 10$ & $48 \pm 6$ & $47 \pm 6$ & $-1.2 \pm 0.2$ & \\
\hline \multirow[t]{3}{*}{ \#40 } & J201836+430018 & $\bar{G}$ & $20: 18: 36.00$ & 43:00:18.0 & 120 & & & & & \\
\hline & & $\mathrm{N}$ & :37.03 & :57.9 & & & & $117 \pm 5$ & & \\
\hline & & S & :34.86 & $42: 59: 33.3$ & & & & $218 \pm 3$ & & \\
\hline \multirow[t]{3}{*}{ \#41 } & J201841+431844 & $\mathrm{Nu}$ & $20: 18: 41.00$ & $43: 18: 44.0$ & 390 & & & $0.8 \pm 0.1$ & & \\
\hline & & $\mathrm{N}$ & $: 40.00$ & $: 22: 04.8$ & & & & $48 \pm 1$ & & \\
\hline & & S & :44.06 & $: 16: 13.2$ & & & & $44 \pm 2$ & & \\
\hline \multirow[t]{3}{*}{ \#42 } & $\mathrm{J} 202220+450835$ & $\mathrm{Nu}$ & $20: 22: 20.01$ & $45: 08: 35.9$ & 60 & & & $0.7 \pm 0.2$ & & \\
\hline & & NW & $: 17.59$ & $: 43.14$ & & & & $8 \pm 1$ & & \\
\hline & & SE & :22.43 & $: 25.6$ & & & & $11 \pm 1$ & & \\
\hline \multirow[t]{3}{*}{ \#43 } & $\mathrm{J} 203755+401328$ & B & $20: 37: 55.00$ & $40: 13: 28.0$ & 60 & & & $7 \pm 1$ & & \\
\hline & & W & :54.16 & :32.6 & & & & $25.7 \pm 2.0$ & & \\
\hline & & $\mathrm{E}$ & $: 57.43$ & $: 29.2$ & & & & $26.5 \pm 2.0$ & & \\
\hline
\end{tabular}




\section{MULTI-WAVELENGTH CROSS-IDENTIFICATION AND ANALYSIS}

Once we have identified and characterised the position and the integrated flux density of the double-lobed source candidates in the radio images, we made use of $\mathrm{NED}^{2}$ and Sim$\mathrm{bad}^{3}$ databases to perform multi-wavelength cross-match positional association at other frequencies. For every doublelobed source, we defined three search regions: one for each lobe and a third for its central point/source/bridge. We consulted the specific catalogues to gather additional information if necessary. In the following subsections, we describe the results.

\subsection{Radio sources}

We found radio counterparts for many of the double-lobed sources candidates. In most cases, the matches belong to the NRAO VLA Sky Survey (NVSS, Condon et al., 1998), performed with the Very Large Array (VLA) at $1.4 \mathrm{GHz}$, with an angular resolution of $45^{\prime \prime}$. Furthermore, many others were reported in surveys carried out with the WSRT, such as the ones mentioned in Sect. 2 by Taylor et al. (1996) and by Setia Gunawan et al. (2003). To highlight the information at $1400 \mathrm{MHz}$ and emphasise the improvement of the angular resolution at $325 \mathrm{MHz}$ that our observations reached, we present, in Figs. A1 to A6, the counterparts found corresponding to the NVSS and WSRT surveys with circles of the size of their attained beams and labelled as '1.4 GHz' and ' $327 \mathrm{MHz}$ ', respectively. Additional detection, reported by previous studies, were also found (but not shown, for the sake of clarity). We will mention them as we describe the counterparts found for each source. For all double-lobed sources with NVSS counterparts, we gathered the NVSS integrated flux density at $1.4 \mathrm{GHz}$, and, using the convolved maps (to a 45" beam) at $610 \mathrm{MHz}$, we derived the spectral index. The results are shown in Table 2.

The spectral index plays an important role in the identification of the dominating process leading to the observed emission. It is well known that negative spectral index values in radio are indicative of a non-thermal emission origin, related to extragalactic sources (e.g. synchrotron, Ginzburg \& Syrovatskii, 1967), but setting a limit is not straight forward around zero values $(0.5<\alpha<-0.5)$ because this range is indicative of optically thin free-free emission (from starforming galaxies or Galactic ionised objects for instance), and also of thick synchrotron emission (core-dominated AGNs). Positive spectral index values can be associated with young compact sources (e.g., Saikia et al., 2004; Pratap \& McIntosh, 2005).

Since our celestial region of study is nearby the Galactic plane, to be conservative, we considered a limiting spectral index value of -0.7 as the main criterion to discriminate

\footnotetext{
${ }^{2}$ https://ned.ipac.caltech.edu/

${ }^{3}$ http://simbad.u-strasbg.fr/simbad/
}

Table 2 Integrated flux density of NVSS counterparts, and spectral indices.

\begin{tabular}{lrrrr}
\hline \hline \# & NVSS ID & $\begin{array}{r}S_{1400 \mathrm{MHz}} \\
(\mathrm{mJy})\end{array}$ & $\begin{array}{r}S C_{610 \mathrm{MHz}} \\
(\mathrm{mJy})\end{array}$ & $\alpha_{1400 \mathrm{MHz}}^{610 \mathrm{MHz}}$ \\
\hline \#6 J201828+402702 & $5.6 \pm 0.7$ & $44 \pm 9$ & $-2.3 \pm 0.3$ \\
\#8 J201910+425044 & $16.6 \pm 0.1$ & $67 \pm 8$ & $-1.7 \pm 0.2$ \\
\#9* J202057+444130 & $109.6 \pm 3.3$ & $353 \pm 98$ & $-1.4 \pm 0.3$ \\
J202057+443959 & & & \\
\#13 J202220+425415 & $71.1 \pm 2.7$ & $279 \pm 45$ & $-1.6 \pm 0.2$ \\
\#17*J202410+432202 & $421 \pm 13$ & $874 \pm 200$ & $-0.9 \pm 0.3$ \\
J202415+432235 & & & \\
\#18 J202422+425729 & $39.6 \pm 1.7$ & $133 \pm 30$ & $-1.4 \pm 0.3$ \\
\#19 J202449+435958 & $15.8 \pm 2.2$ & $42 \pm 8$ & $-1.1 \pm 0.3$ \\
\#20 J202625+442056 & $12 \pm 0.7$ & $41 \pm 8$ & $-1.5 \pm 0.2$ \\
\#21 J202634+412952 & $5.7 \pm 0.8$ & $81 \pm 3$ & $-3.2 \pm 0.2$ \\
\#24 J202754+423205 & $124.7 \pm 4.3$ & $402 \pm 74$ & $-1.4 \pm 0.2$ \\
\#25 J202816+442100 & $5 \pm 0.7$ & $20 \pm 3$ & $-1.7 \pm 0.2$ \\
\#28 J202943+425119 & $7.5 \pm 0.7$ & $23 \pm 5$ & $-1.3 \pm 0.3$ \\
\#29 J203201+413722 & $15.2 \pm 0.8$ & $151 \pm 20$ & $-2.8 \pm 0.3$ \\
\#30 J203323+412723 & $8.4 \pm 0.7$ & $96 \pm 21$ & $-2.9 \pm 0.2$ \\
\#32 J203402+420614 & $11.6 \pm 0.7$ & $119 \pm 20$ & $-2.8 \pm 0.3$ \\
\#35 J203431+413046 & $27.4 \pm 1.5$ & $40 \pm 10$ & $-0.4 \pm 0.2$ \\
\#38 J203555+421803 & $604 \pm 21$ & $2546 \pm 359$ & $-1.7 \pm 0.3$ \\
\#39 J203720+405924 & $22.3 \pm 3.2$ & $48 \pm 7$ & $-0.8 \pm 0.2$ \\
\#42 J202219+450837 & $11 \pm 2$ & $20 \pm 3$ & $-0.7 \pm 0.3$ \\
\#43 J203756+401326 & $8.7 \pm 0.7$ & $65 \pm 15$ & $-2.4 \pm 0.3$ \\
\hline
\end{tabular}

The spectral indices were derived between catalogued NVSS integrated flux densities $(S)$ and the convolved integrated flux densities $(S C)$ of the double-lobed sources at $610 \mathrm{MHz}$. *In order to estimate the total spectral index we added the NVSS integrated flux densities of both lobes.

the double-lobed source candidates between extragalactic $(\alpha \leq-0.7)$ or Galactic $(\alpha>-0.7)$ origin (see, for instance, Ibar et al., 2010; Ocran et al., 2020). Whenever was possible, we did also consider the value of the spectral index $\alpha_{1400 \mathrm{MHz}}^{610 \mathrm{MHz}}$ in our classification. The detailed information of the flux densities measured and the spectral indices derived are given in Tables $1 \& 2$.

\subsection{Sources at other wavelengths}

Although NED and Simbad databases cover the entire electromagnetic spectrum, besides radio sources, only infrared ones were found, except for one X-ray counterpart. Infrared sources are expected to be frequent in the line of sight towards the Cygnus region. But we note that as double radio sources are likely extragalactic objects, and the Cygnus OB2 region is in the Galactic plane, it is remarkably challenging to find conclusive evidence of the right counterparts in optical/IR for these radio sources.

Even though we defined three search regions, as is explained at the beginning of the section, we focused the infrared search on the surroundings of the centre $(\mathrm{Nu})$ or the geometric centre $(\mathrm{G})$ of the double-lobed sources, 
considering that we aim to identify the possible host galaxy for the proposed extragalactic sources. In checking for the positional overlapping in the plane of the sky, we considered the following extensions of infrared sources for each catalogue using circles with diameters of: 6 "for WISE (Wright et al., 2010), 2" for 2MASS (Skrutskie et al., 2006), 2" for Spitzer (Werner et al., 2004), and 18" for IRAS (Neugebauer et al., 1984). Below, we present fully the arguments that led us to the classification of each double-lobed source.

\subsection{Extragalactic sources}

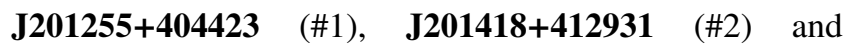
$\mathbf{J 2 0 1 8 4 7 + 4 0 1 2 3 0}(\# 7)$. At the position of these sources there are no detection at other frequencies, see Fig. A1. An inclination of the line linking the lobes towards the line of sight, will result in a modification of the actual sizes/shapes as seen in the images. In particular, an inclination angle smaller than 45 deg will imply higher projection effects, including the appreciation of lobes with different sizes. We interpret that this is the case for source \#1.

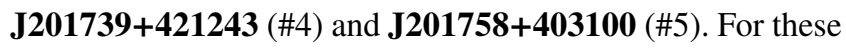
sources we found no associations at other frequencies, see Fig. A1. As a complement, we show the spectral index maps as well as the error distribution in Fig. B1.

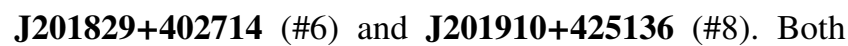
sources have positional coincidences with NVSS catalogued records, see Table 2. For \#8, the NVSS component overlaps only the southern lobe (NVSS ID: J201910+425044). The radio counterpart of \#6, which is a component of the NVSS survey is not resolved, see Fig. A1.

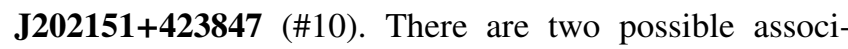
ations at infrared wavelengths for this source: WISEA J202150.84+423846.9 and 2MASS J20215131+4238521, coincident with the position of the geometric centre. Besides, the radio image at $610 \mathrm{MHz}$ reveals two knots in-between the IR sources and the lobes (see Figs. A2 and C1). These knots could be part of common bipolar jets (an unseen weak bridge) formed time ago, of an AGN with much activity in the past. The morphology could be also indicative of DDRG, as two double radio galaxies that share a centre.

J202209+415033 (\#11), J202218+411728 (\#12), $\mathbf{J 2 0 2 2 2 2 + 4 2 5 4 0 9}(\# 13)$ and $\mathbf{J 2 0 2 3 1 5 + 4 3 0 2 5 2}$ (\#14). For these sources, there are no detection at other wavelengths, except for \#13, which has one radio counterpart, unresolved, at $1.4 \mathrm{GHz}$, see Fig. A2 and Table 2.

$\mathbf{J 2 0 2 3 5 9 + 4 3 5 5 2 5}$ (\#15). There is one possible association at infrared wavelengths (WISEA J202358.42+435519.3), in agreement with the position of the nucleus, see Fig. A2 and
Fig. C1. It might be revealing the location of the putative host galaxy. We show the spectral index distribution and error maps; see Fig. B1.

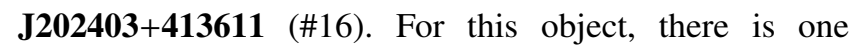
possible association at infrared frequencies (WISEA J202403.03+413611.3), at its geometric centre; see Figs. A2 and $\mathrm{C} 1$. Considering the spectral index value within the errors, the object might be an extra-galactic source where the core may have reignited, giving it a similar brightness as the southern lobe.

J202413+432220 (\#17). This source was detected by previous radio surveys: at $365 \mathrm{MHz}$ with the TEXAS telescope (Douglas et al., 1996b, $S=1.76 \pm 0.14 \mathrm{Jy}, 4^{\prime}$ beam), and at $5 \mathrm{GHz}$ with the MIT-green Bank telescope (Bennett et al., 1986, $S=69 \pm 11 \mathrm{mJy}$, beam of $3^{\prime}$ ). We found two NVSS components coincident with each lobe (NVSS IDs: J202410+432202, J202415+432235; see Fig. A2 and Table 2.

J202422+425722 (\#18), J202448+435956 (\#19) and J202625+442048 (\#20). Each of these sources have coincident NVSS components, all unresolved (see Figs. A2, A3 and Table 2).

J202632+412942 (\#21) and J202755+423215 (\#24). For both sources there are possible associations at the infrared band, WISEA J202634.96+412943.8 and WISEA J202755.97+423224.3, respectively. Besides, the two sources are also unresolved NVSS components. Moreover, the source \#21 has positional coincidence with a WSRTGP catalogue record; see Figs. A3 and C1.

J202645+431435 (\#22). There is one possible association at infrared frequencies for this source (WISEA J202645.01+431439.8), which could be pinpointing the host galaxy, see Figs. A3 and C1. The morphology of this source seems to indicate that the nucleus is closer to the southern lobe.

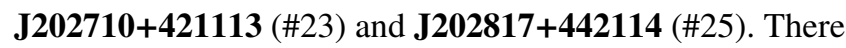
are just one detection at other frequencies for \#25, for which there is an NVSS component overlapping its southern lobe (NVSS ID: J202816+442100), Fig. A3.

J202919+441341 (\#27). There are no associations at other wavelengths. The intensity distribution at both radio bands is complex. We may be looking at a double-lobed source, where projection effects play an important role, see Fig. A3. The possibility that we were looking at just one lobe (to the north) and that the southern component is the core seems unlikely, since this latter source do not present the flat spectral index expected from cores.

$\mathbf{J 2 0 2 9 4 3 + 4 2 5 1 2 2}$ (\#28). There is one counterpart at radio frequencies. It is reported in the NVSS catalogue (NVSS ID: 
J202943+425119), see Fig. A4 and Table 2; the source is unresolved.

J203201+413730 (\#29). For this source, there are several counterparts at radio frequencies. Is also known as NVSS J203201+413722 and is unresolved. Martí et al. (2007) studied it in detail; they proposed it as a radio galaxy which might suffered different episodes of jet activity (see Fig. A4). The authors observed the field using the VLA $\mathrm{C}+\mathrm{D}$ array configuration at $5 \mathrm{GHz}$ as well as the GMRT at $610 \mathrm{MHz}$. These observations, along with a marginal Chandra X-ray detection in the vicinity of \#29 reported by Butt et al. (2007), made it a radio galaxy candidate with an optically-thick compact core (Martí et al., 2007). The two lobes of \#29 were also reported by Setia Gunawan et al. (2003) at their two observing bands; they named them as SBHW-217 $\left(S_{350 \mathrm{MHz}}=85 \pm 5 \mathrm{mJy}, S C_{1400 \mathrm{MHz}}=36 \pm 5 \mathrm{mJy}\right)$ -northern lobe- and SBHW-218 $\left(S_{350 \mathrm{MHz}}=122 \pm 35 \mathrm{mJy}\right.$, $S C_{1400 \mathrm{MHz}}=39 \pm 5 \mathrm{mJy}$ ) -southern lobe-, where the quoted integrated fluxes $S C$ at $1400 \mathrm{MHz}$ were derived from images convolved to the $325 \mathrm{MHz}$ beam.

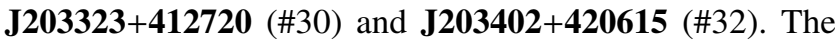
source \#30 was previously reported by Martí et al. (2007) and is also an unresolved NVSS component. The source \#32 has one infrared counterpart (WISEA J203401.86+420618.2), and a radio counterpart which is an unresolved NVSS component, see Fig. A4 and Fig. C1.

$\mathbf{J 2 0 3 3 4 5 + 4 0 4 0 1 5 ~ ( \# 3 1 ) . ~ T h e r e ~ i s ~ o n e ~ p o s s i b l e ~ a s s o c i - ~}$ ation at infrared frequencies, with the object 2MASS $\mathrm{J} 20334540+4040133$. At radio frequencies, the NW lobe was reported in WSTRGP (Taylor et al., 1996) and both lobes were reported by Setia Gunawan et al. (2003), at two bands; the ones here called NW, as SBHW-106 $\left(S_{350 \mathrm{MHz}}=75 \pm 12 \mathrm{mJy}, S C_{1400 \mathrm{MHz}}=25 \pm 4 \mathrm{mJy}\right)$, and SE, as SBHW-104 $\left(S_{350 \mathrm{MHz}}=82 \pm 4 \mathrm{mJy}, S C_{1400 \mathrm{MHz}}=34 \pm 7 \mathrm{mJy}\right)$; see Figs. A4 and C1. As a complement we show the spectral index map as well as the error maps in Fig. B1.

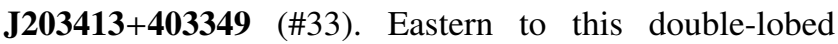
source there is a third source ( 40" apart, and $30^{\prime \prime}$ in size); Setia Gunawan et al. (2003) reported $1.4 \mathrm{GHz}$ WSRT emission at its position, from data with angular resolution of $13^{\prime \prime}$. We measured $S_{325 \mathrm{MHz}}=134 \pm 16 \mathrm{mJy}$ and $S C_{610 \mathrm{MHz}}=68 \pm 8 \mathrm{mJy}$ for this third source; the spectral index is then $\alpha_{325}^{610}=-1.7 \pm 0.3$. The angular resolutions at the three bands $(325,610$ and $1400 \mathrm{MHz})$ are sufficient to clearly detach the three sources; besides, the northern and southern lobes present extension towards each other, challenging a physical association between this eastern source and the double-lobed \#33. See Fig. A4.

$\mathbf{J 2 0 3 4 2 5}+\mathbf{4 0 4 6 0 5}$ (\#34) and $\mathbf{J 2 0 3 4 3 1}+\mathbf{4 0 3 3 3 2}(\# 36)$. We found no detections at other frequencies for these sources. See Fig. A4.

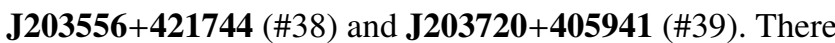
are counterparts at radio frequencies. Both are reported in the NVSS catalogue. Nevertheless, from the NVSS cutouts, only \#39 is resolved at $1.4 \mathrm{GHz}$. The source \#38 has a radio counterpart, which is a component of the WSRTGP surveys (Condon et al., 1998; Taylor et al., 1996) and also one X-ray possible counterpart (SSTSL2 J203557.22+421744.0, Evans et al., 2010). See Fig. A5.

$\mathbf{J 2 0 1 8 3 6}+\mathbf{4 3 0 0 1 8}(\# 40)$ and $\mathbf{J 2 0 2 2 2 0}+\mathbf{4 5 0 8 3 5}(\# 42)$. There are possible associations at infrared frequencies for both sources; one for \#40 (WISEA J201836.53+430018.7) and two for \#42 (2MASS J20221985+4508338 and WISEA J202219.75+450834.5). Besides, \#42 was also reported as an NVSS component, the source is unresolved. See Figs. A6 and C2.

$\mathbf{J 2 0 3 7 5 5}+\mathbf{4 0 1 3 2 8}(\# 43)$. There is one counterpart at radio frequencies. The source is listed in the NVSS catalogue, it is unresolved. See Fig. A6.

\subsection{Galactic sources}

In the sample of double-lobed sources studied here, there are a few with flat or positive spectrum, for which non-thermal radiation mechanisms do not apply. Their emission can be explained advocating thermal processes, by close-by (Galactic) plasma media, that emit either under optically thick or thin regimes (Osterbrock, 1989).

J202859+402141 (\#26). This source shows one possible counterpart, nearby the geometric centre and at infrared frequencies (WISEA J202858.96+402146.8), and another at radio frequencies (WSRTGP component); See Figs. A3 and $\mathrm{C} 1$. The spectral index of the northern lobe is close to zero, while that of the southern lobe is $>1$, see Table 1 . We note that the spectral index error values are high, because the signal-to-noise at the $325 \mathrm{MHz}$ image was low at the position of this source. However, both indices are consistent with those corresponding to Galactic emission. Due to that, we propose that \#26 belongs to the Galaxy.

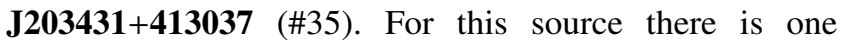
infrared detection onto the bridge, coincident with the proposed nucleus, WISEA J203433.70+412953.7 and one counterpart at radio frequencies (NVSS component, ID: J203431+413046, unresolved);see Figs. A4 and C2. Besides, there is an HII region at $R A, \operatorname{Dec}(\mathbf{J} 2000)=20: 34: 31.9$, $+41: 30: 52$ (G080.522+00.714, Anderson et al., 2015). The spectral index suggests that it is a Galactic object. 


\subsection{Dubious cases}

Besides the (37+2) double-lobed sources already identified either as extragalactic or Galactic, there remain four cases in which the true nature is not straightforward to unveil, and are analysed below.

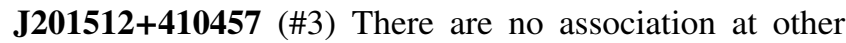
frequencies for this source; see Fig. A1. The radio image at $610 \mathrm{MHz}$ shows that the components present a kind of extension towards each other. Taking this into account, and the fact that the spectral index of both components (similar values) is below -0.7 , we favour an extragalactic origin for this object.

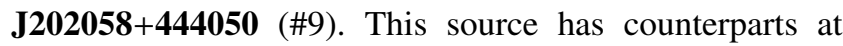
radio frequencies. Each lobe candidate is coincident with a NVSS component; see Fig. A1. The northern one presents a negative spectral index $(-0.7 \pm 0.1)$, while the spectral index of the southern one is close to -0.1 . This led us to propose that instead of a double-lobed source, it could well be two different objects, an extragalactic (northern) one and the other of Galactic nature. An alternate possibility is that the southern component is a radio core, of which only the northern lobe is being detected. The sizes of each object are 45 and 20 arcsec, north and south respectively, separated by a distance of 2 arcmin. The evidence gathered is not enough to confirm this candidate as a double-lobed source.

J203529+403201 (\#37). There are no detections at other frequencies at its position; see Fig. A5.The spectral indices of both lobes, separated $45 \mathrm{arcsec}$, are quite different; in particular the one associated with SW lobe, which is close to zero, see Table 1. A possible scenario as an extragalactic source, is that the SW component is a bright hotspot, with its flat-spectrum emission being the reason why the spectral index is so different from the NE component.

$\mathbf{J 2 0 1 8 4 1 + 4 3 1 8 4 4}$ (\#41) shows emission that resembles two highly collimated jets extending along 7 arcmin on the sky. It presents a knot in between the two lobes, indicative of a possible nucleus. The source is not covered by our $325 \mathrm{MHz}$ mosaic image and lacks of infrared or radio putative counterparts; see Fig. A6. Notwithstanding, the morphology of the object is typical AGN-like sources.

\section{DISCUSSION}

\subsection{Generalities on spectral indices}

Thirty-nine out of the 43 candidates presented in Table 1 were observed at the two GMRT bands (325 and $610 \mathrm{MHz}$ ), allowing them to retrieve information on their spectral indices. We plotted the layout of those 39 sources in the RA-Dec sky, including a spectral index scale (Fig. 2). No trends are recognised, along a $\sim 5 \times 5 \mathrm{sq}$ deg area $\left(0^{\circ} \leq b<5^{\circ}\right)$, both with regards to the indices and the location of the sources

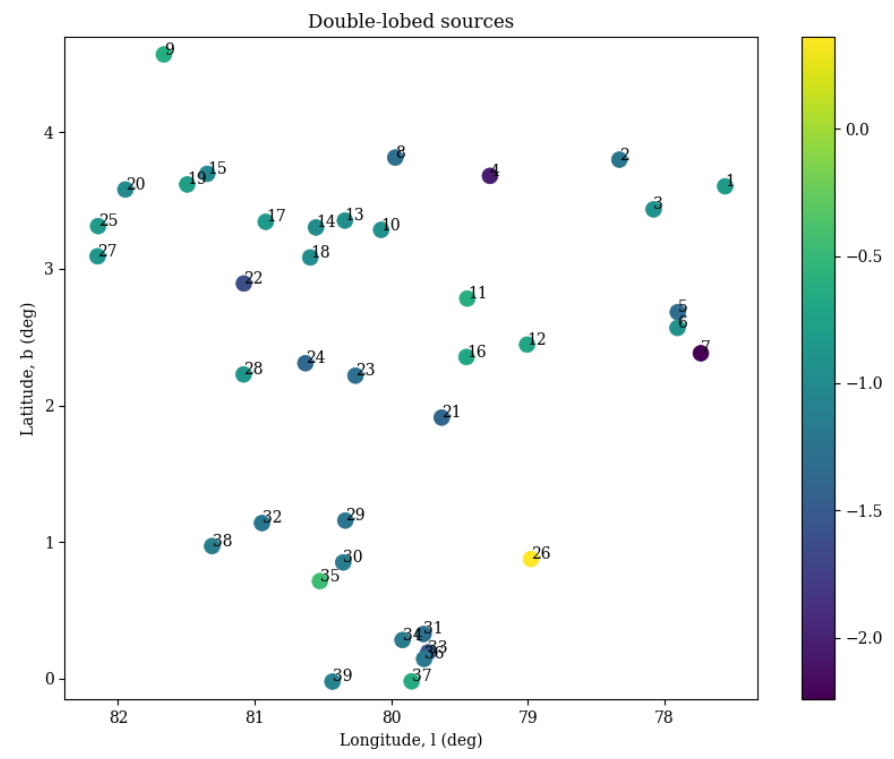

Figure 2. Layout of the observed double-lobed sources in Galactic coordinates, with their spectral indices represented with colours, according to the scale at the right.

relative to the Galactic plane.

The total spectral index values, derived using the integrated flux density along the whole candidate including material in-between lobes, remain in the interval $[-2.5,+0.5]$ (see Fig. 3). The median spectral index value is $-1.0 \pm 0.1$. This is in line with the fact that at the observed bands, the processes involved in non-thermal emission make sources brighter than those related to thermal sources, especially in the case of non-extended ones. In addition, the strategy followed when imaging the original mosaics was to use a weighting scheme aimed to outline discrete sources against diffuse or extended emission.

For those (among the more extended) sources with a high signal-to-noise ratio $(>21)$, we built the spectral index distribution maps; they are shown in Fig. B1. The higher $\alpha$ values in the central region are indicative of the nucleus of an extragalactic source.

For completeness, we present in Fig. 4 the distribution of the total sizes of the 43 candidates (Table 1), as seen in the $610 \mathrm{MHz}$ band.

\subsection{Recap on cross-identifications}

The cross-identification of the 43 candidates with previous surveys such as the NVSS contributed to examining each candidate's nature, and helped as verification of the detections presented here (see Sect. 4).

We found fourteen IR detections overlapping the nucleus or proposed geometric centre of twelve radio candidates reported along Sect. 4. Out of these twelve positional coincidences, nine were only WISE sources, one was a 2 MASS, and for the remaining two, we found sources in both the WISE and 2MASS catalogues. The search criteria for the po- 


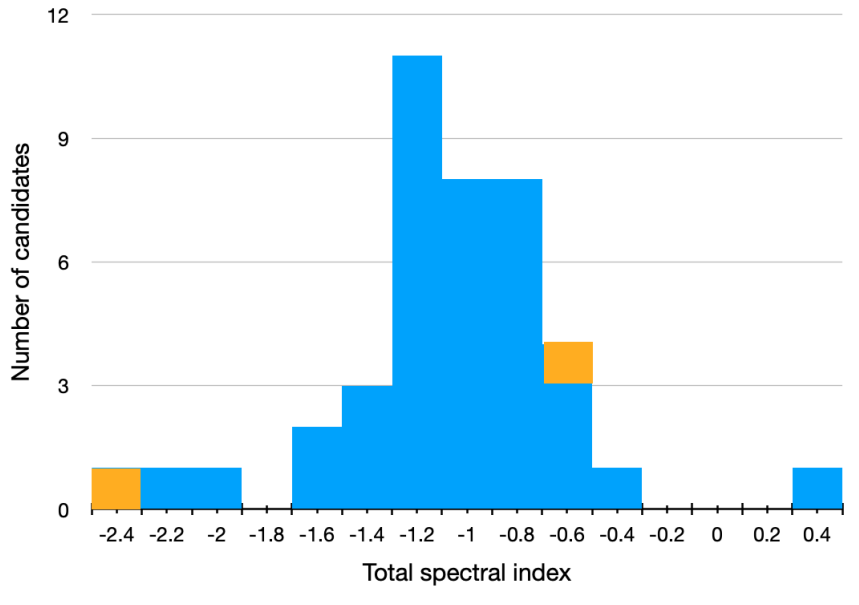

Figure 3. Number of candidates with spectral index information, as a function of their total spectral index $\left(\alpha_{\mathrm{T}}\right)$ between $325 \mathrm{MHz}$ and $610 \mathrm{MHz}$, for the 39 double-lobed detected at both frequency bands with the GMRT (in blue), and for the sources \#42, \#43 between $610 \mathrm{MHz}$ and $1400 \mathrm{MHz}$ (in orange).

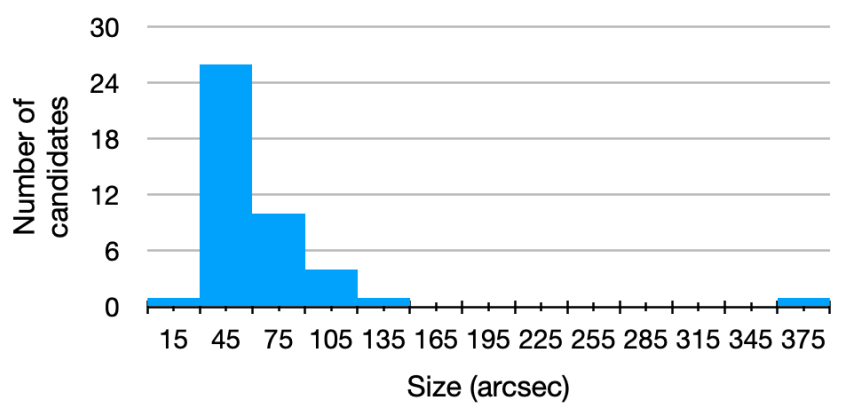

Figure 4. Distribution of sizes of the 43 double-lobed candidates. The extension of the sources ranged from $\sim 25 \operatorname{arcsec}$ up to $\sim 375 \operatorname{arcsec}(\sim$ 7 arcmins). sitional overlapping is given Sect. 4.2. The location of those IR sources are shown in the radio images (Figs. A1 to A6), labelled as 'WISE' and '2MASS'. We present in Figs. C1 and $\mathrm{C} 2$ the infrared images towards the mentioned eleven candidate. We superposed the 325 and $610 \mathrm{MHz}$ emission contours, which allowed us to explore the association between the IR sources and the double-lobed candidates.

The X-ray counterpart found favours the extragalactic origin of source \#38, and might indicate that this source is at a closer distance than the rest, see e.g. Elvis et al. (1978); Masetti et al. (2013); Lanzuisi et al. (2013) and references therein.

\subsection{The extragalactic double-lobed sources}

After the careful inspection of the 43 double-lobed source candidates presented in this work, and taking into account all the information collected, we found that 37 of them are compatible with an extragalactic nature. For those sources that we proposed a WISE source as a possible candidate, which could be associated with the host galaxy, we estimated the WISE colours W1-W2 and W2-W3, following Gürkan et al. (2014). The values that we obtained (W1-W2 $<0.2$ and W2-W3 $<5.8)$ are compatible with those shown by radioloud AGNs (see Fig. 3 in Gürkan et al., 2014). Among the 37 sources we found some of them with peculiar morphology such as:

- \#19, \#30 and \#34, that resemble head-tail galaxies like the ones compiled by Pal \& Kumari (2021), see for example their Fig. 2.

- \#29, which seems to be of FRI type.

\subsection{On ultra steep spectrum sources}

The existence a radio counterpart at $1.4 \mathrm{GHz}$ allowed us to test for Ultra-Steep Spectrum sources (USS), i.e., objects with $\alpha \leq-1.3$. Saxena et al. (2018) searched USS as proxies of high-redshift radio galaxies (HzRGs), for which they request additional characteristics. The authors identified HzRG through a radio colour-colour plot (sources with $\alpha_{1400}^{610}<-1.5$, see their figure 5), the absence of optically identified counterparts and angular sizes smaller than $30^{\prime \prime}$ (see also references therein). Then, following Saxena et al. (2018), and for the sources detected at both GMRT bands that possess a radio counterpart at $1.4 \mathrm{GHz}$, we built the radio colour-colour diagram $\alpha_{325}^{610}$ vs $\alpha_{1400}^{610}$; see Fig. 5. The plot reveals that the majority of the double-lobed sources have a constant or a steep spectrum. In particular, there are five sources at the loci of USS ones, with $\alpha<<-1.5$ : \#6, $\# 21, \# 29, \# 30$ and \#32. It can be appreciated in Table 2 that these five sources, together with \#43, are the ones with the steepest spectral indices, $\alpha \leq-2.3$; \#43 was not covered by our $325 \mathrm{MHz}$ image and, it will not be analysed. Only one of the sources has an extension of 30" (source \#6). New 


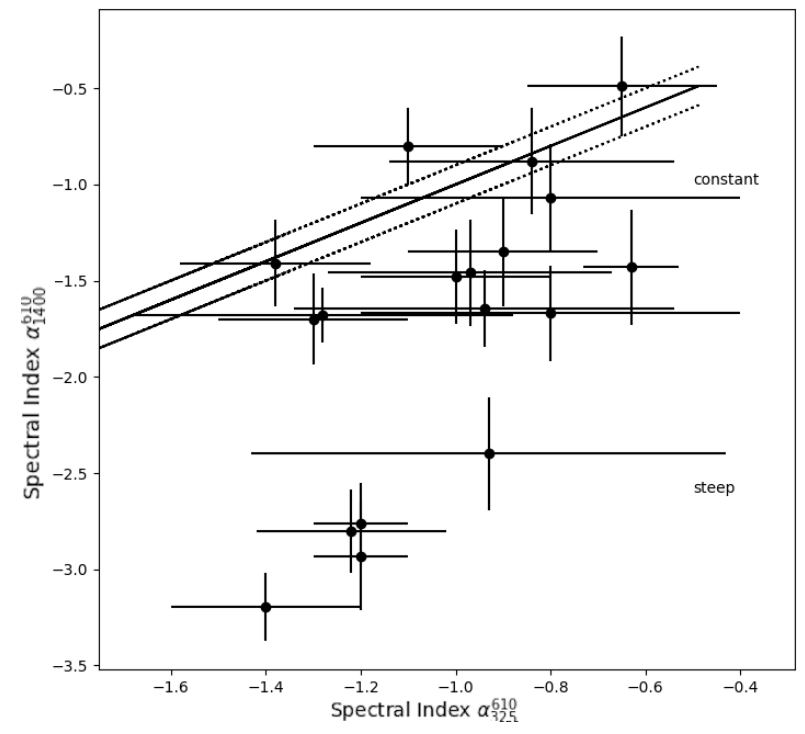

Figure 5. Radio colour-colour diagram. The spectral indices of the detected double-lobed sources between 325 and $610 \mathrm{MHz}$ versus the spectral indices between 1400 and $610 \mathrm{MHz}$. The solid and dashed lines mark the constant spectral index at both radio colours and the adopted errors of 0.1 . The sources with $\alpha_{1400}^{610}<<-1.5$ are considered to be ultra-steep-spectrum sources.

observations at $1.4 \mathrm{GHz}$, with better sensitivity and higher angular resolution, are needed to confirm whether candidate \#6 is a USS source.

\section{STATISTICS AND CONCLUSIONS}

- In a region of $19.7 \mathrm{sq}$ deg of the sky, 43 probable doublelobed sources were found at $610 \mathrm{MHz}, 39$ of them also at $325 \mathrm{MHz}$.

- The angular resolutions of the data sets were $6^{\prime \prime}$ to $10^{\prime \prime}$, and the average sensitivities, 0.2 and $0.5 \mathrm{mJy} \mathrm{beam}^{-1}$ (at 610 and $325 \mathrm{MHz}$, respectively).

- The extension of the sources ranged from $\sim 25$ arcsec up to $\sim 7$ arcmins.

- Twenty-one out of the 43 sources have a bridge.

- Eleven out of the 43 sources have a detected nucleus.

- Thirty-seven out of the 43 sources have spectral indices characteristic of extragalactic objects, probably AGNs.

- Nine out of the 37 proposed as extragalactic sources present infrared sources at the position of the a putative nucleus.

- Two out of the 43 sources have flat or positive spectral indices, favouring a Galactic origin.

- For one of the 43 sources, the two emitting objects are probably not physically related.

- Three out of the 43 remains as dubious cases, though with feature(s) compatible with an extragalactic nature.

- The median spectral index value of the extragalactic sources is $-1.0 \pm 0.1$. Five extragalactic sources present an ultra steep spectra. Only one of them (\#6) has a small size $\left(\leq 30^{\prime \prime}\right)$ consistent with a high-redshift radio galaxy.
- Although the large angular scale probed at the highest observing band was $17^{\prime}$, no sources with extension above 7 arcmin were detected.

\section{ACKNOWLEDGEMENTS}

The radio data presented here were obtained with the Giant Metrewave Radio Telescope (GMRT). The GMRT is operated by the National Centre for Radio Astrophysics of the Tata Institute of Fundamental Research. We thank the staff of the GMRT that made these observations possible. This research has made use of the NASA/IPAC extragalactic database (NED) that is operated by the Jet Propulsion Laboratory, California Institute of Technology, under contract with the National Aeronautics and Space Administration and also we made use of the SIMBAD database, operated at CDS, Strasbourg, France, and of NASA's Astrophysics Data System bibliographic series. J.S. and P.B. acknowledge support from ANPCyT PICT 0773-2017. The authors want to thank the anonymous referee for the careful reading of the manuscript and their many insightful comments and suggestions. C.H.I.C. acknowledges the support of the Department of Atomic Energy, Government of India, under the project 12-R\&D-TFR-5.02-0700.

\section{A APPENDIX}

In this appendix we present the GMRT images of 39 doublelobed source candidates at 610 and $325 \mathrm{MHz}$, and the remaining 4 -out of a total of 43- only observed at $610 \mathrm{MHz}$ (Figs. A1 to A6). The candidates showed radio emission at rather different scales. The one with the lowest $\sigma$ value is $\# 12\left(\sigma_{12}=0.1 \mathrm{mJy}_{\text {beam }}{ }^{-1}\right)$. To allow comparison among sub-figures, we added in the bottom right corner of each one a scale number (preceded with an ' $x$ '), that represents the factor to multiply $\sigma_{12}$ to get the $\sigma$ of the candidate sub-figure.

For some of the candidates (\#4, \#5, \#15,\#31) we show the spectral index distribution and error maps (Fig. B1). And for those probable galaxies with putative infrared counterparts we present the radio contours on top of the IR images (Figs. C1 and $\mathrm{C} 2$ ).

\section{REFERENCES}

Albacete Colombo J. F., Caramazza M., Flaccomio E., Micela G., Sciortino S., 2007, VizieR Online Data Catalog, pp $\mathrm{J} / \mathrm{A}+\mathrm{A} / 474 / 495$

Anderson L. D., Armentrout W. P., Johnstone B. M., Bania T. M., Balser D. S., Wenger T. V., Cunningham V., 2015, ApJS, 221, 26

Anglada G., Rodríguez L. F., Carrasco-González C., 2018, A\&A Rev., 26, 3

Benaglia P., De Becker M., Ishwara-Chandra C. H., Intema H. T., Isequilla N. L., 2020a, PASA, 37, e030

Benaglia P., Ishwara-Chandra C. H., Intema H., Colazo M. E., Gaikwad M., 2020b, A\&A, 642, A136

Benaglia P., Ishwara-Chandra C. H., Paredes J. M., Intema H. T., Colazo M. E., Isequilla N. L., 2021, ApJS, 252, 17 

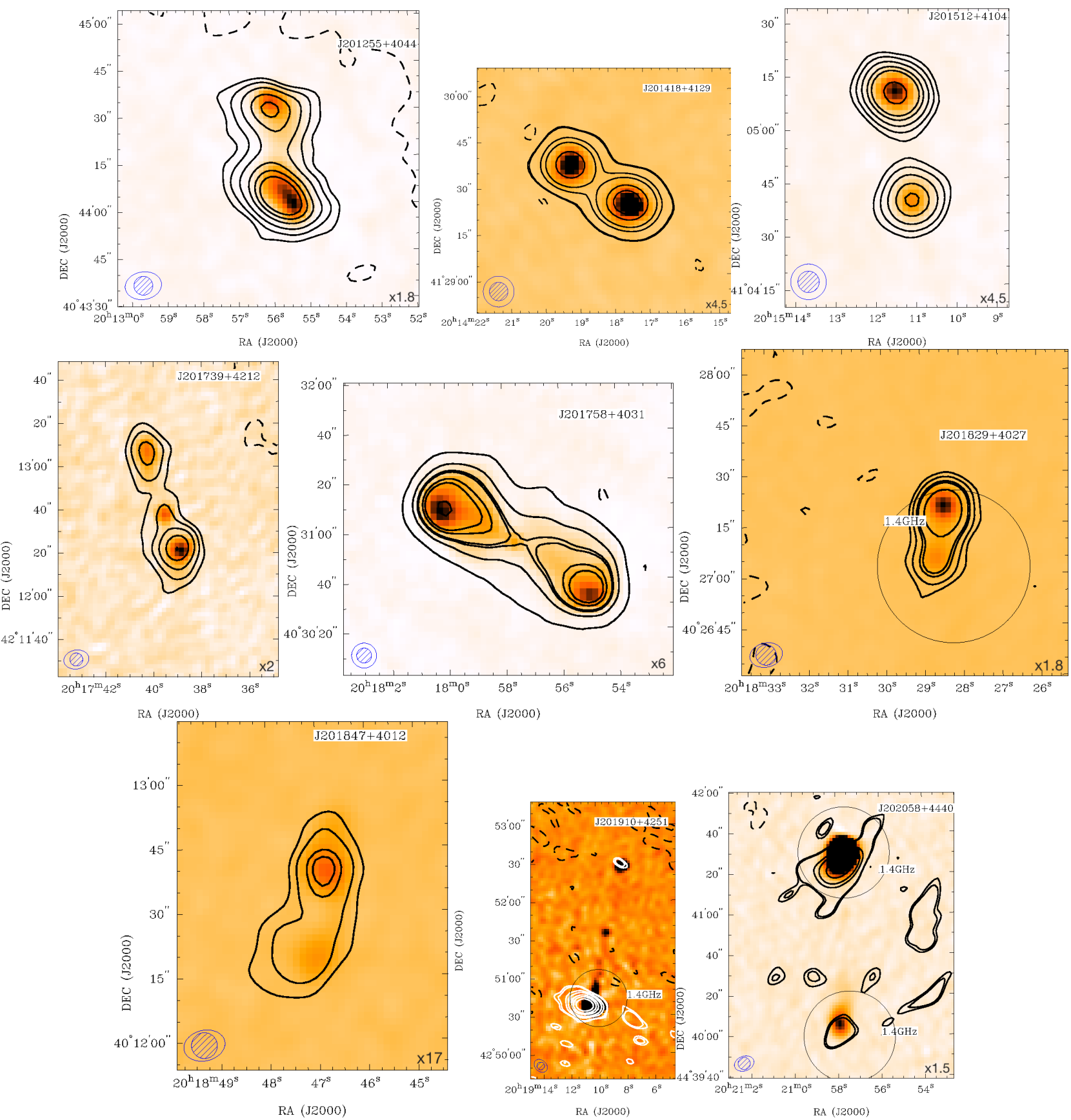

RA (J2000)

RA (J2000)
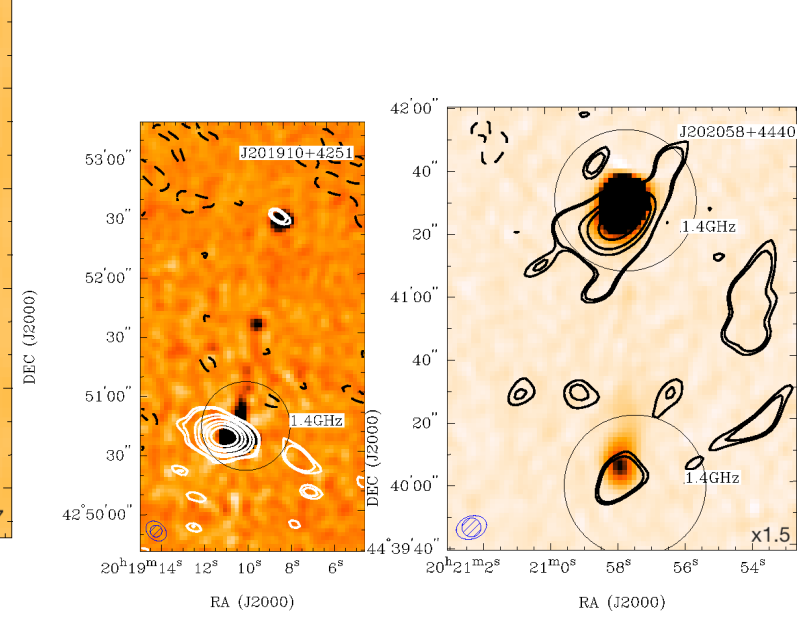

Figure A1. GMRT images of the double-lobed sources at $610 \mathrm{MHz}$ (colour scale) and $325 \mathrm{MHz}$ (contours). The contour levels and colour scale interval for each source are as follows: \#1-J201255+404423: $-2.5,2.5,3,15,27,60$ and 90, in units of $\left.\sigma\left(=0.18 \mathrm{mJy}_{\text {beam }}{ }^{-1}\right) ; 1.6,20\right] \mathrm{mJy}^{-1}$ beam ${ }^{-1}$. \#2-J201418+412931: $-2.5,2.5,3,15,27,60$, and 90 , in units of $\sigma\left(=0.45\right.$ mJy beam $\left.^{-1}\right) ;[3,13]$ mJy beam $^{-1}$.\#3-J201512+410457: $-2.5,2.5,5,9,15,21$, and 41 , in units of $\sigma\left(=0.45 \mathrm{mJy}_{\text {beam }}{ }^{-1}\right) ;[4,11] \mathrm{mJy}_{\text {beam }}{ }^{-1}$.\#4-J201739+421243: $-2.5,2.5,5,9,21$, and 27, in units of $\sigma\left(=0.2 \mathrm{mJy} \mathrm{beam}^{-1}\right) ;[0.4,4] \mathrm{mJy} \mathrm{beam}^{-1}$.\#5$\mathrm{J} 201758+403100:-2.5,2.5,15,27,30,45,55$, and 100, in units of $\sigma\left(=0.6 \mathrm{mJy}_{\text {beam }}{ }^{-1}\right) ;[0.6,17] \mathrm{mJy}$ beam $^{-1}$. \#6-J201828+402714: $-2.5,2.5,3,15,27$, 60, and 90, in units of $\sigma\left(=0.18 \mathrm{mJy}_{\text {beam }}^{-1}\right) ;[6,21] \mathrm{mJy} \mathrm{beam}^{-1}$. \#7-J201847+401230: $-2.5,2.5,5,9,11$, and 21, in units of $\sigma\left(=1.7 \mathrm{mJy} \mathrm{beam}^{-1}\right)$; [6, 21] $\mathrm{mJy}_{\text {beam }}{ }^{-1}$. \#8-J201910+425136: $-2.5,2.5,3,6,9,15,21$, and 30, in units of $\sigma\left(=0.7 \mathrm{mJy}\right.$ beam $\left.{ }^{-1}\right) ;[0.7,9] \mathrm{mJy} \mathrm{beam}^{-1}$. \#9-J202058+444050: -2.5 , $2.5,3,15,27,60$, and 90 , in units of $\sigma\left(=0.15 \mathrm{mJy} \mathrm{beam}^{-1}\right) ;[0.7,9] \mathrm{mJy}^{-1} \mathrm{bem}^{-1}$. The synthesised beams are shown at the bottom-left corner of each image. NVSS detections are marked with a $45^{\prime \prime}$ ' diameter circle and labelled as ' $1.4 \mathrm{GHz}$ '. For the $\mathrm{x}$ factor of the bottom-right corner, see text. 

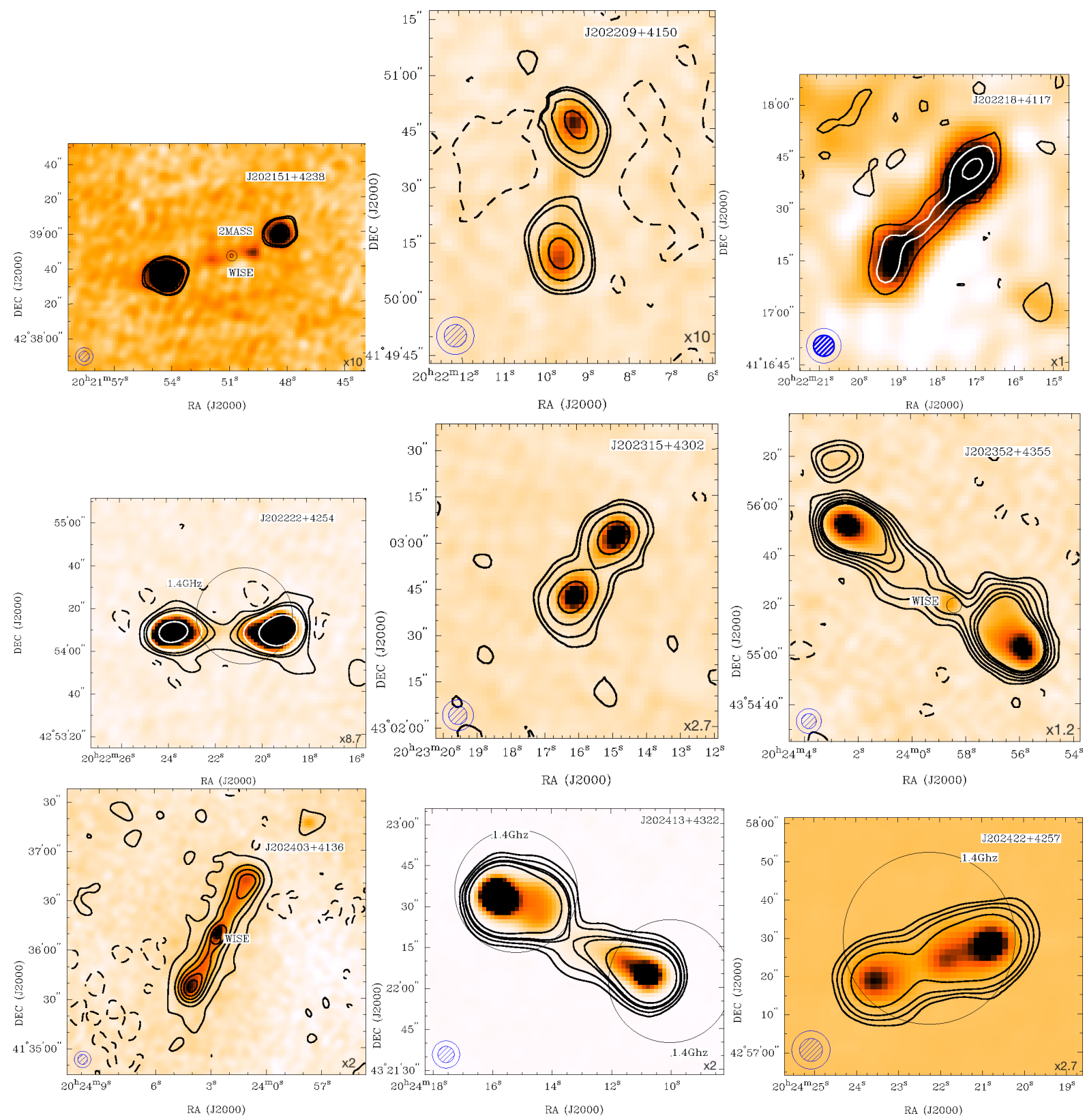

Figure A2. GMRT images of the double-lobed sources at $610 \mathrm{MHz}$ (colour scale) and $325 \mathrm{MHz}$ (contours). The contour levels and colour scale interval for

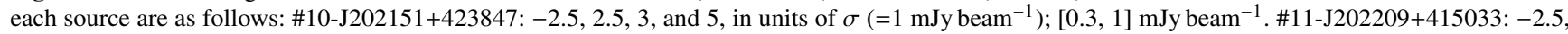
$2.5,3,5$, and 15, in units of $\sigma\left(=1 \mathrm{mJy} \mathrm{beam}^{-1}\right) ;[0.4,3.9] \mathrm{mJy} \mathrm{beam}^{-1}$. \#12-J202218+411728: $-2.5,2.5,10$, and 30, in units of $\sigma\left(=0.1 \mathrm{mJy}^{-1}\right.$ beam $\left.{ }^{-1}\right)$;

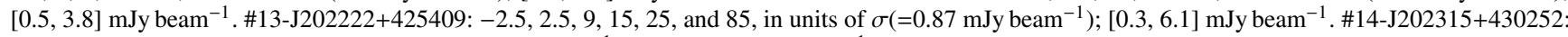
$-2.5,2.5,9,15$, and 25 , in units of $\sigma\left(=0.27 \mathrm{mJy} \mathrm{beam}^{-1}\right) ;[0.3,7.9] \mathrm{mJy}_{\text {beam }}{ }^{-1}$. \#15-J202359+435525: $-2.5,2.5,5,9,15,21$, and 27, in units of

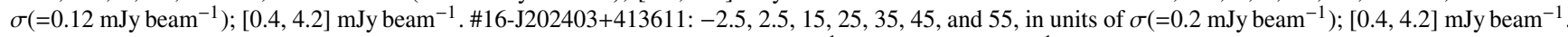
$\# 17-\mathrm{J} 202413+432220:-2.5,2.5,5,15,21$, and 30 , in units of $\sigma\left(=0.2 \mathrm{mJy}_{\text {beam }}^{-1} ;[1,4.4]\right.$ mJy beam $^{-1}$. \#18-J202422 $+425722:-2.5,2.5,5,15$, and 21, in units of $\sigma\left(=0.27 \mathrm{mJy}_{\text {beam }}^{-1}\right) ;[6,21] \mathrm{mJy}^{-1} \mathrm{bem}^{-1}$. The synthesised beams are shown at the bottom-left corner of each image. NVSS detections are marked with a 45" diameter circle and labelled as '1.4 GHz'. WISE and 2MASS sources are marked and labelled as well.For the $\mathrm{x}$ factor of the bottom-right corner, see text. 

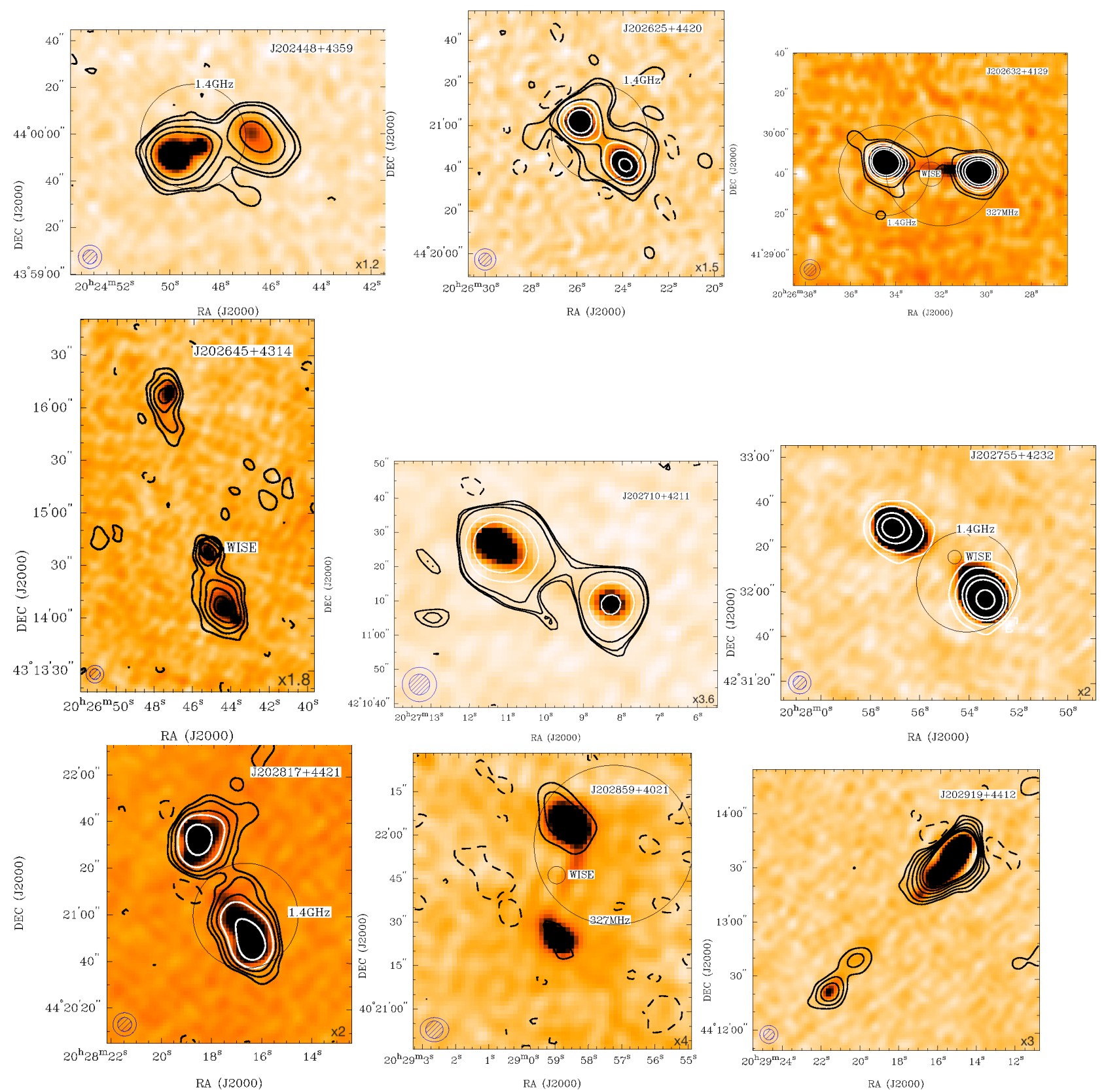

Figure A3. GMRT images of the double-lobed sources at $610 \mathrm{MHz}$ (colour scale) and $325 \mathrm{MHz}$ (contours). The contour levels and colour scale interval for each source are as follows: \#19-J202448+435956: $-2.5,2.5,5,10$, and 27, in units of $\sigma\left(=0.12 \mathrm{mJy}_{\text {beam }}{ }^{-1}\right)$; $[0.3,2.6] \mathrm{mJy}^{2}$ beam $^{-1}$. \#20-J202625+442048: $-2.5,2.5,5,25,35,75$, and 135, in units of $\sigma\left(=0.15 \mathrm{mJy}_{\text {beam }}{ }^{-1}\right) ;[0.3,2] \mathrm{mJy}_{\text {beam }}{ }^{-1}$. \#21-J202632+412942: $-2.5,2.5,5,10,15,25$, and 35, in units of $\sigma\left(=1 \mathrm{mJy}_{\text {beam }}^{-1}\right) ;[0.3,0.6] \mathrm{mJy}_{\text {beam }}{ }^{-1}$. \#22-J202645+431435: $-2.5,2.5,5,9,15,21$, and 27, in units of $\sigma\left(=0.18 \mathrm{mJy} \mathrm{beam}^{-1}\right) ;[0.2,0.7] \mathrm{mJy}^{2}$ beam ${ }^{-1}$. \#23-J202710+421113: $-2.5,2.5,3,5,15$, and 35, in units of $\sigma\left(=0.36 \mathrm{mJy} \mathrm{beam}^{-1}\right) ;[0.3,3.9] \mathrm{mJy}_{\text {beam }}{ }^{-1}$. \#24-J202755+423215: $-2.5,2.5,30,50,130$, and 310 , in units of $\sigma\left(=0.2 \mathrm{mJy}_{\text {beam }}{ }^{-1}\right) ;[0.3,3.9] \mathrm{mJy} \mathrm{beam}^{-1}$. \#25-J202817+442114: $-2.5,2.5,5,10,15,35$, and 85, in units of $\sigma\left(=0.16 \mathrm{mJy} \mathrm{beam}^{-1}\right) ;[0.8$, 1] $\mathrm{mJy}_{\text {beam }}{ }^{-1}$. \#26-J202859+402141: $-2.5,2.5,5,9,15,21$, and 27, in units of $\sigma\left(=0.4 \mathrm{mJy} \mathrm{beam}^{-1}\right) ;[0.6,1.8] \mathrm{mJy} \mathrm{beam}^{-1}$. \#27-J202919+441341 -2.5 , $2.5,5,9,15,21$, and 27 , in units of $\sigma\left(=0.3 \mathrm{mJy}_{\text {beam }}{ }^{-1}\right) ;[0.4,2] \mathrm{mJy}_{\text {beam }}{ }^{-1}$. The synthesised beams are shown at the bottom-left corner of each image. NVSS and WSRT detections are marked and labelled as ' $1.4 \mathrm{GHz}$ ' and '327 MHz'. WISE and 2MASS sources are marked and labelled as well. For the $\mathrm{x}$ factor of the bottom-right corner, see text. 

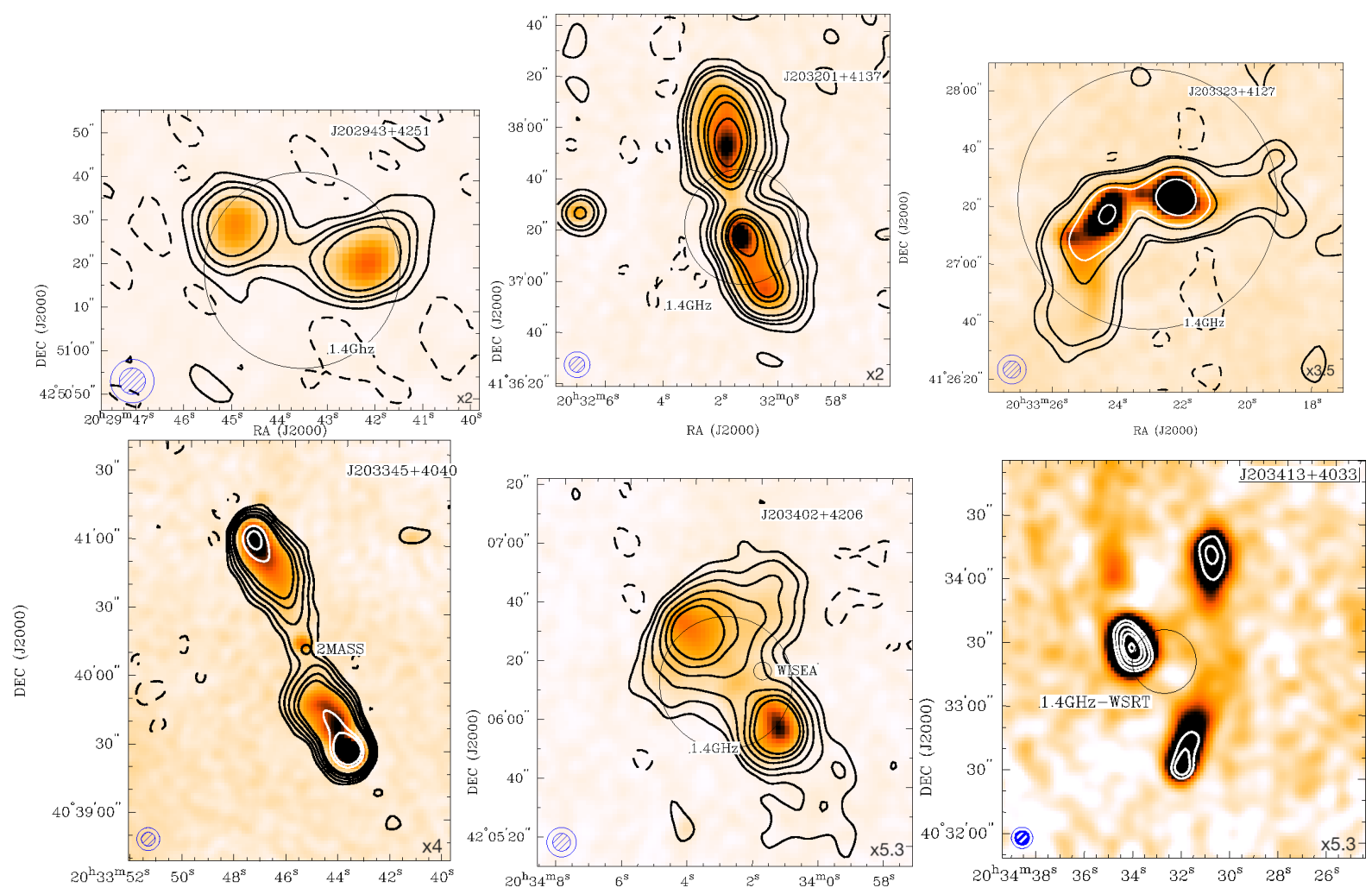

RA (J2000)
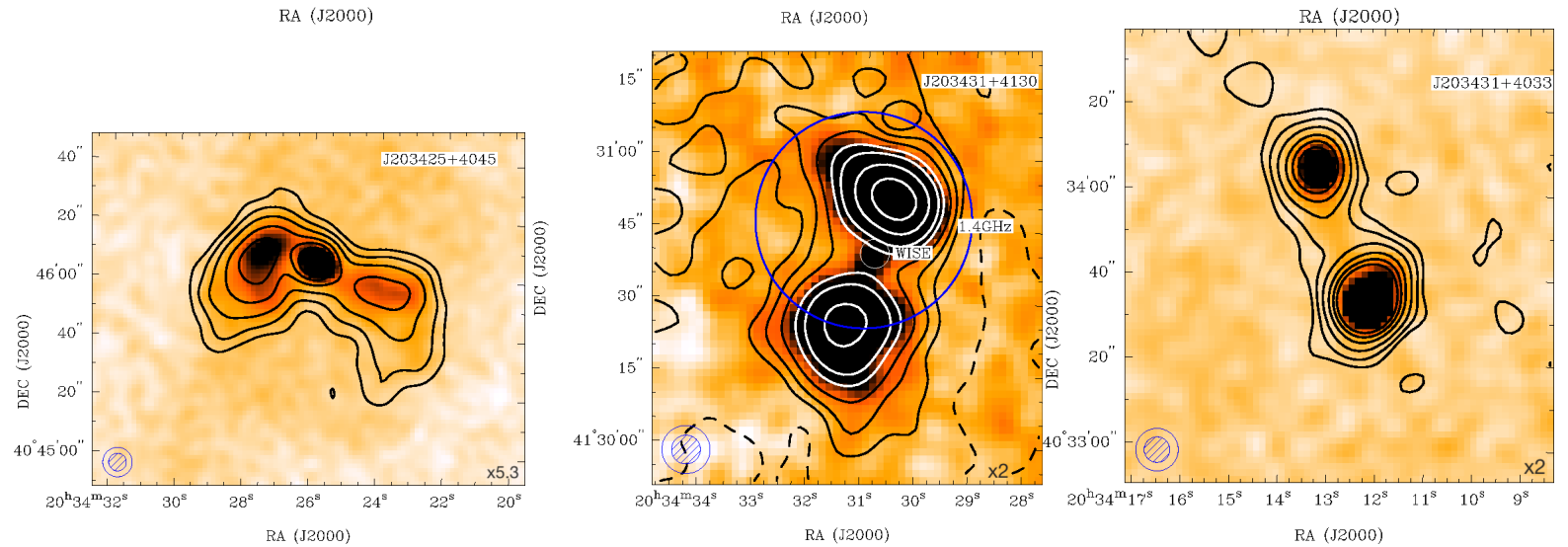

Figure A4. GMRT images of the double-lobed sources at $610 \mathrm{MHz}$ (colour scale) and $325 \mathrm{MHz}$ (contours). The contour levels and colour scale interval for each source are as follows: \#28-J202943+425122: $[-2.5,2.5,9,15,27]$ in units of $\sigma\left(=0.2 \mathrm{mJy}_{\text {beam }}{ }^{-1}\right) ;[0.4,1] \mathrm{mJy}^{2}$ beam ${ }^{-1}$. \#29-J203201+413730: -2.5 , $2.5,9,15,27,57,87,107$, and 150 , in units of $\sigma\left(=0.2 \mathrm{mJy} \mathrm{beam}^{-1}\right) ;[0.4,1] \mathrm{mJy}_{\text {beam }}{ }^{-1}$. \#30-J203323+412720: $-2.5,2.5,5,27,40$, and 100, in units of $\sigma\left(=0.35 \mathrm{mJy}_{\text {beam }}^{-1}\right) ;[0.6,5] \mathrm{mJy}_{\text {beam }}^{-1}$. \#31-J203345+404015: $-2.5,2.5,5,9,15,21,55$, and 75, in units of $\sigma\left(=0.4 \mathrm{mJy} \mathrm{beam}^{-1}\right) ;[0.5,5] \mathrm{mJy}^{-1}$ beam ${ }^{-1}$ \#32-J203402+420615: $-2.5,2.5,5,9,15,21$, and 27, in units of $\sigma\left(=0.53\right.$ mJy beam $\left.^{-1}\right) ;[0.4,10]$ mJy beam $^{-1}$. \#33-J203413+403349: $-2.5,2.5,5,9,15,21$, and 27, in units of $\sigma\left(=0.53 \mathrm{mJy}_{\text {beam }}^{-1}\right) ;[0.4,10] \mathrm{mJy}_{\text {beam }}^{-1}$. \#34-J203425+404605: $-2.5,2.5,5,9,15,21$, and 27, in units of $\sigma\left(=0.53 \mathrm{mJy}^{2}\right.$ beam $\left.{ }^{-1}\right) ;[0.3$,

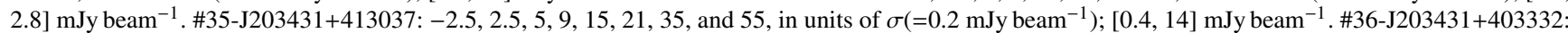
$-2.5,2.5,5,9,15,21$, and 27, in units of $\sigma\left(=0.53 \mathrm{mJy}^{\text {beam }}{ }^{-1}\right) ;[0.2,0.7] \mathrm{mJy}^{-1}$ beam ${ }^{-1}$. The synthesised beams are shown at the bottom-left corner of each image. NVSS and WSRT beams detection are marked with a circles and labelled as ' $1.4 \mathrm{GHz}$ ' and '1.4GHz-WSRT'. WISE and 2MASS position errors are marked with circles and labelled as well. For the $\mathrm{x}$ factor of the bottom-right corner, see text. 

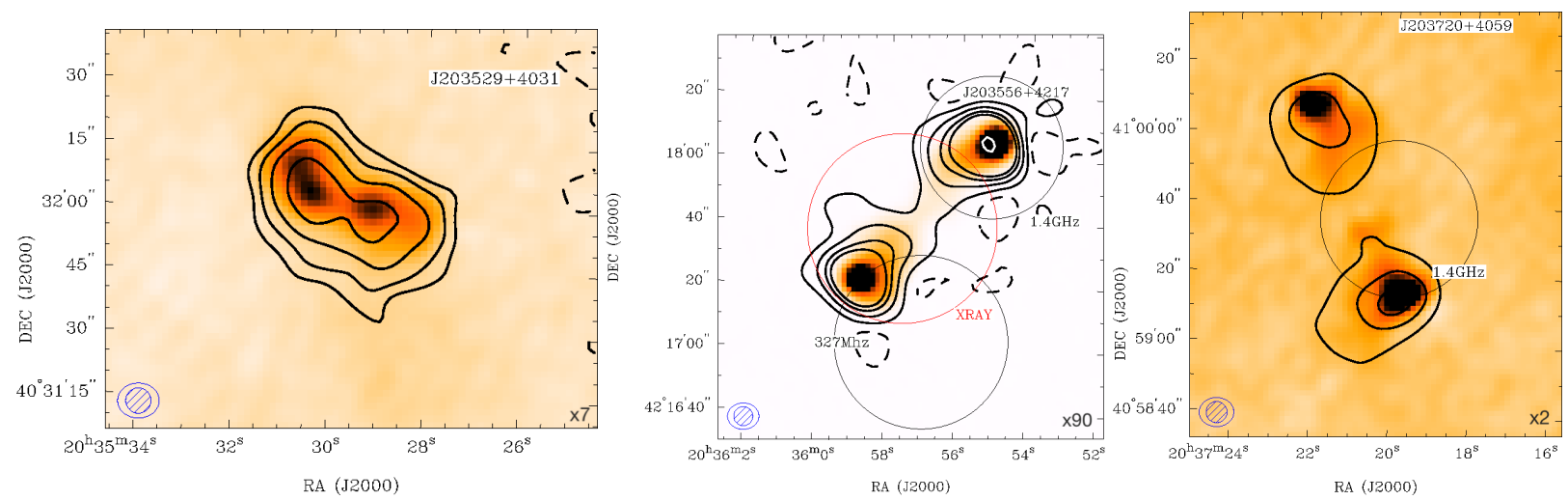

Figure A5. GMRT images of the double-lobed sources at $610 \mathrm{MHz}$ (colour scale) and $325 \mathrm{MHz}$ (contours). The contour levels and colour scale interval for each source are as follows: \#37-J203529+403201: $-2.5,2.5,5,9,15,21$, and 27, in units of $\sigma\left(=0.7 \mathrm{mJy}_{\text {beam }}^{-1}\right) ;[1,8] \mathrm{mJy} \mathrm{beam}^{-1}$. \#38-J203556+421744: $-2.5,2.5,10,20,30,120,200,300$, and 500, in units of $\sigma\left(=9 \mathrm{mJy} \mathrm{beam}^{-1}\right) ;[3,237] \mathrm{mJy} \mathrm{beam}^{-1}$. \#39-J203720+405941: $-2.5,2.5$, and 5, in units of $\sigma\left(=0.2 \mathrm{mJy}_{\text {beam }}{ }^{-1}\right) ;[2,7] \mathrm{mJy}_{\text {beam }}{ }^{-1}$. The synthesised beams are shown at the bottom-left corner of each image. NVSS beam detection is marked with a circle and labelled as ' $1.4 \mathrm{GHz}$ ' while the $\mathrm{X}$-ray detection is shown with a red circle. For the $\mathrm{x}$ factor of the bottom-right corner, see text.
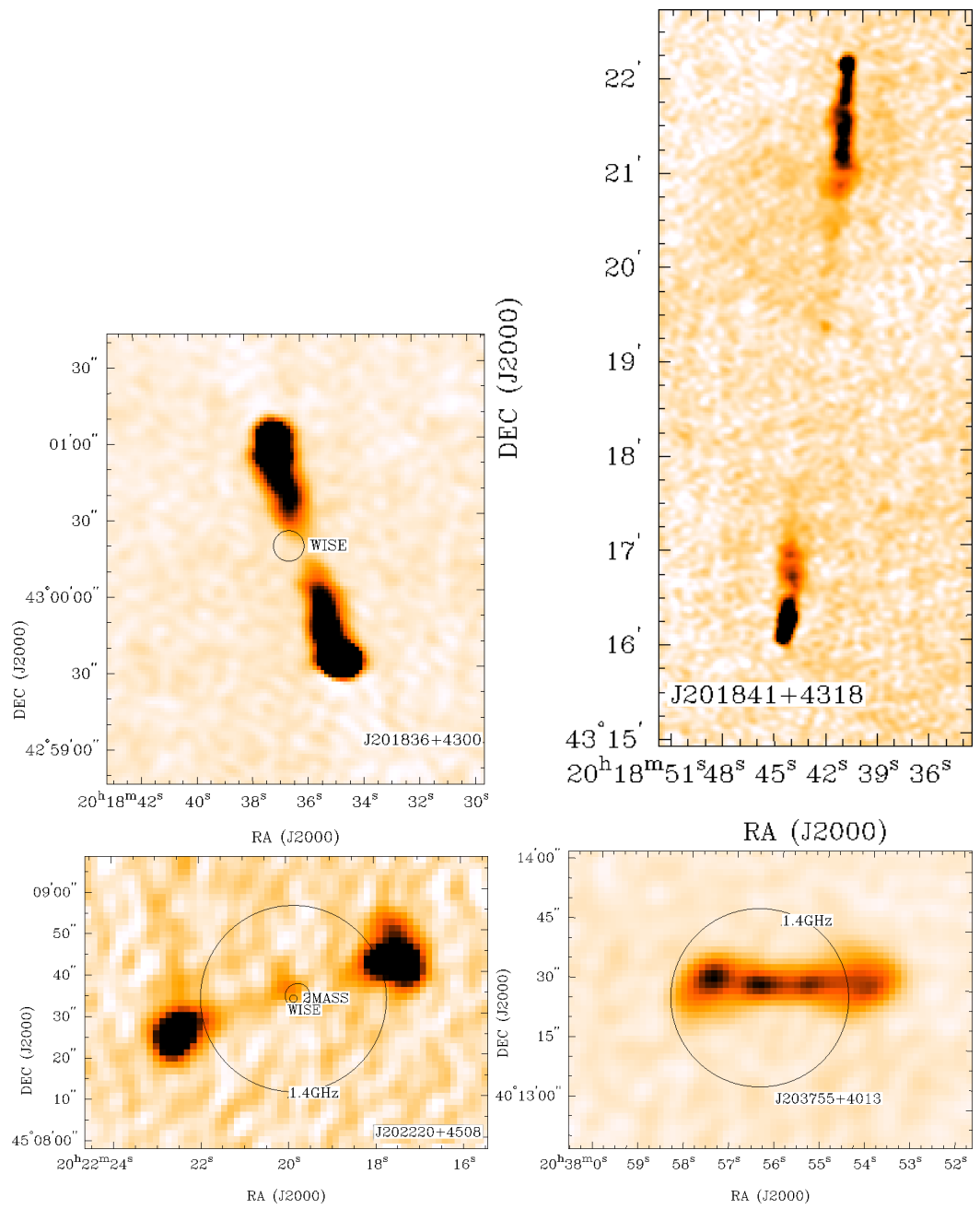

Figure A6. GMRT images of the double-lobed sources at $610 \mathrm{MHz}$ (colour scale). NVSS beam detection is marked with a circle and labelled as ' $1.4 \mathrm{GHz}$ '. WISE and 2MASS position errors are marked with circles and labelled as well. The sources and colour scale interval represented are: \#40-J201836+430018: [0.4, 5] mJy beam ${ }^{-1}$; \#41-J201841+431844: [0.2, 1.6] mJy beam $^{-1}$; \#42-J202220+450835: [0.4, 5.8] mJy beam ${ }^{-1}$; \#43-J203755+401328: [0.4, 5.8] mJy beam ${ }^{-1}$. 

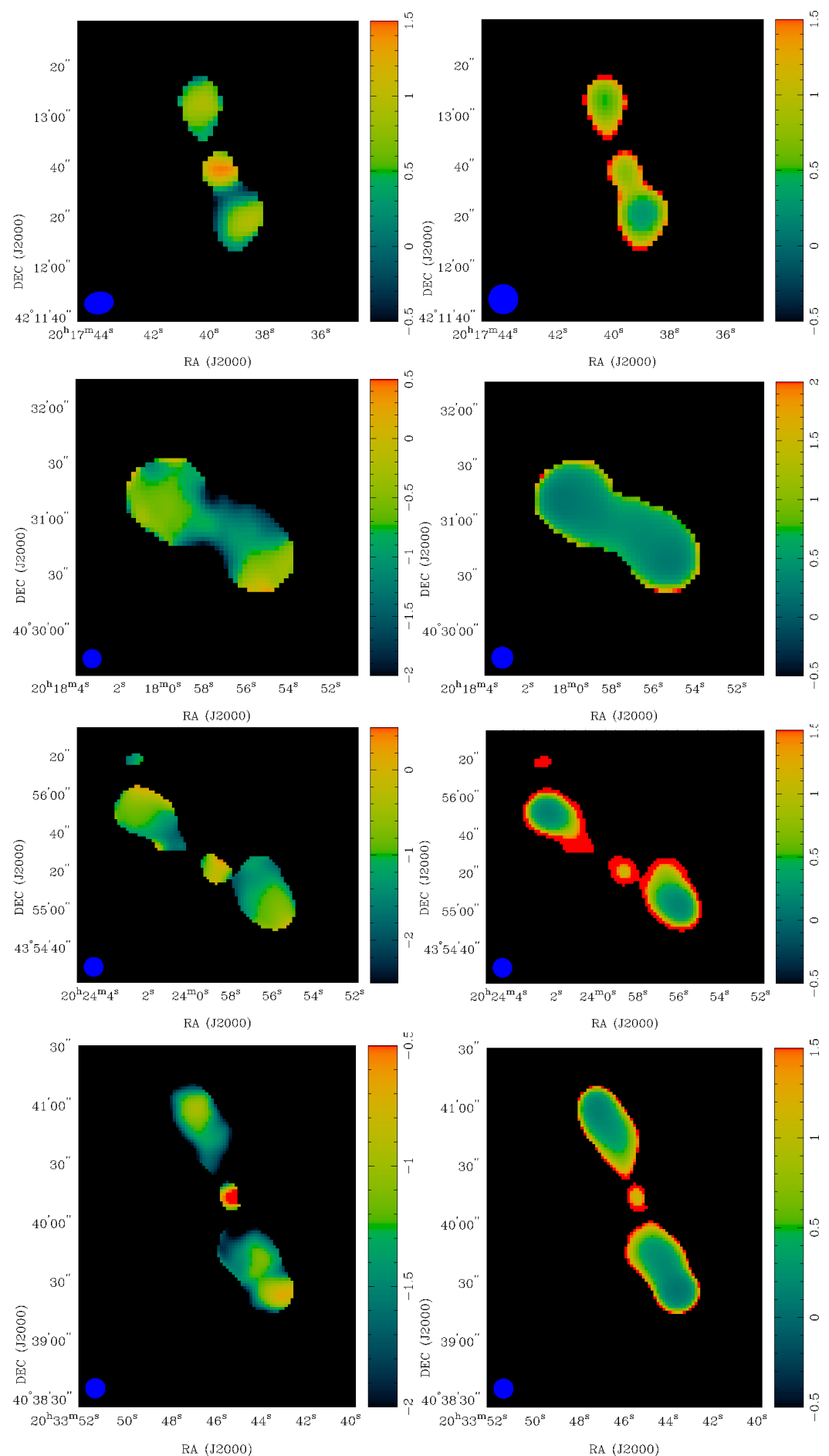

Figure B1. Spectral index (left) and error maps (right) of J201739+421252 (\#4, first row), J201758+403100 (\#5, (second row), J202359+435525 (\#15, third row), and J203345+404015 (\#31, fourth row) sources. The synthesised beam is shown at the bottom-left corner of each image. 

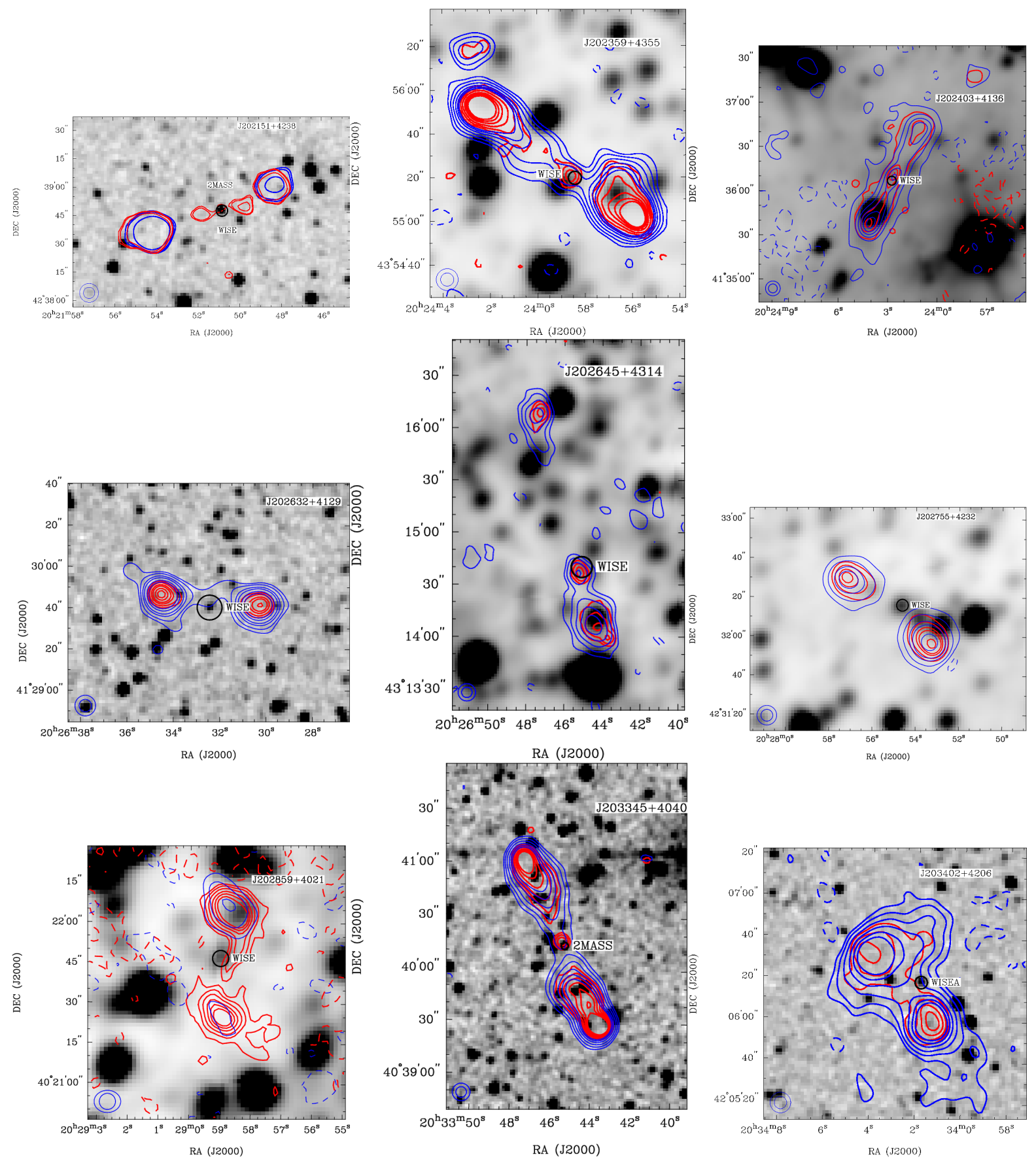

Figure C1. GMRT contours at $610 \mathrm{MHz}$ (red) and $325 \mathrm{MHz}$ (blue) overlaid on WISE (W1-band)/2MASS (3.4 $\mu \mathrm{m}$ ) infrared images. The contour levels at $325 \mathrm{MHz}$ are the same shown in Figs. A1-A6. The contour levels at $610 \mathrm{MHz}$ are: \#10-J202151+423847: $-2.5,2.5,3$, and 5, in units of $\sigma\left(=0.13\right.$ mJy beam $\left.^{-1}\right)$. \#15-J202352+435525: $-2.5,2.5,5,9,15,21$, and 27, in units of $\sigma\left(=0.1\right.$ mJy beam $\left.^{-1}\right)$. \#16-J202403+413611: $-2.5,2.5,5$, and 15 , in units of $\sigma\left(=0.15 \mathrm{mJy}\right.$ beam $\left.^{-1}\right)$. \#21-J202632+412942: $-2.5,2.5,5,10,15,25$, and 35, in units of $\sigma\left(=0.1 \mathrm{mJy} \mathrm{beam}^{-1}\right)$. \#22-J202645+431435: $-2.5,2.5,5,9$, and 15, in units of $\sigma\left(=0.1 \mathrm{mJy}\right.$ beam $\left.^{-1}\right)$. \#24-J202755+423215: $-2.5,2.5,30,50$, and 130, in units of $\sigma\left(=0.1 \mathrm{mJy} \mathrm{beam}^{-1}\right)$. \#26-J202859+402141: $-2.5,2.5$, $9,15,21$, and 27, in units of $\sigma\left(=0.1 \mathrm{mJy}\right.$ beam $\left.^{-1}\right)$. \#31-J203345+404015: $-2.5,2.5,9,15,21$, and 27, in units of $\sigma\left(=0.2 \mathrm{mJy} \mathrm{beam}^{-1}\right)$. \#32-J203402+420615: $-2.5,2.5,9,15,21$, and 27 , in units of $\sigma\left(=0.1 \mathrm{mJy}_{\text {beam }}^{-1}\right)$. The synthesised beams are shown at the bottom-left corner of each image. 

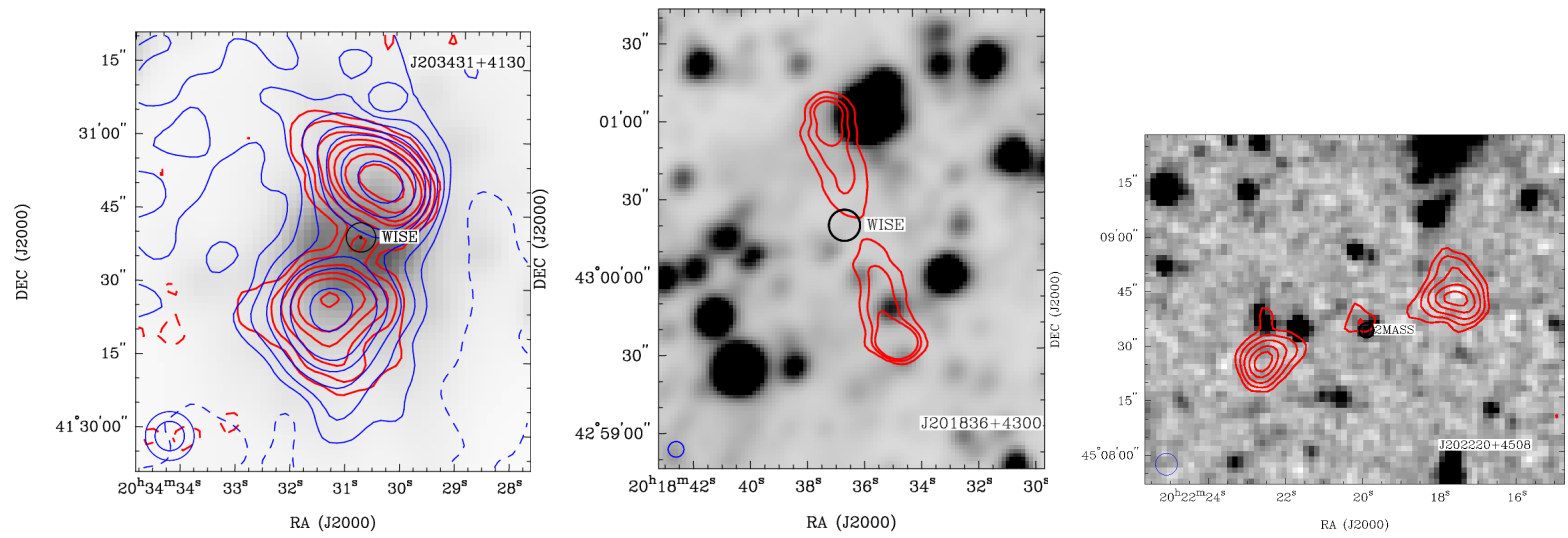

Figure C2. GMRT contours at $610 \mathrm{MHz}$ (red) and $325 \mathrm{MHz}$ (blue) overlaid on WISE/2MASS infrared images. The contour levels at $325 \mathrm{MHz}$ are the same shown in Figs. A1-A6. The contour levels at $610 \mathrm{MHz}$ are: \#35-J203431+413037: $-2.5,2.5,9,15,21,35$, and 55 , in units of $\sigma\left(=0.12 \mathrm{mJy}^{-1}\right.$ beam $\left.{ }^{-1}\right)$. \#40-J201836+430018: $-2.5,2.5,10$, and 20, in units of $\sigma\left(=0.5\right.$ mJy beam $\left.^{-1}\right)$. \#42-J203431+413035: $-2.5,2.5,5,9,15,21,35,55$, and 85, in units of $\sigma\left(=0.15 \mathrm{mJy}_{\text {beam }}{ }^{-1}\right)$. The synthesised beams are shown at the bottom-left corner of each image.

Bennett C. L., Lawrence C. R., Burke B. F., Hewitt J. N., Mahoney J., 1986, ApJS, 61, 1

Brocksopp C., Kaiser C. R., Schoenmakers A. P., de Bruyn A. G., 2011, MNRAS, 410, 484

Brown M. J. I., Duncan K. J., Landt H., Kirk M., Ricci C., Kamraj N., Salvato M., Ananna T., 2019, MNRAS, 489, 3351

Butt Y. M., Combi J. A., Drake J., Finley J. P., Konopelko A., Lister M., Rodriguez J., Shepherd D., 2007, in Ritz S., Michelson P., Meegan C. A., eds, American Institute of Physics Conference Series Vol. 921, The First GLAST Symposium. pp 429-430 (arXiv: astro-ph/0703017), doi:10.1063/1.2757386

Carrasco-González C., Rodríguez L. F., Anglada G., Martí J., Torrelles J. M., Osorio M., 2010, Science, 330, 1209

Condon J. J., Cotton W. D., Greisen E. W., Yin Q. F., Perley R. A., Taylor G. B., Broderick J. J., 1998, AJ, 115, 1693

Douglas J. N., Bash F. N., Bozyan F. A., Torrence G. W., Wolfe C., 1996a, VizieR Online Data Catalog, p. VIII/42

Douglas J. N., Bash F. N., Bozyan F. A., Torrence G. W., Wolfe C., 1996b, AJ, 111, 1945

Elvis M., Maccacaro T., Wilson A. S., Ward M. J., Penston M. V., Fosbury R. A. E., Perola G. C., 1978, MNRAS, 183, 129

Evans I. N., et al., 2010, ApJS, 189, 37

Fanaroff B. L., Riley J. M., 1974, MNRAS, 167, 31P

Ginzburg V. L., Syrovatskii S. I., 1967, in International Cosmic Ray Conference. p. 48

Greisen E. W., 2003, AIPS, the VLA, and the VLBA. ., p. 109, doi:10.1007/0-306-48080-8_7

Gürkan G., Hardcastle M. J., Jarvis M. J., 2014, MNRAS, 438,1149

Ibar E., Ivison R. J., Best P. N., Coppin K., Pope A., Smail I., Dunlop J. S., 2010, MNRAS, 401, L53

Intema H. T., 2014, in Astronomical Society of India Confer- ence Series. p. 469 (arXiv : 1402 . 4889)

Isequilla N. L., Fernández-López M., Benaglia P., IshwaraChandra C. H., del Palacio S., 2019, A\&A, 627, A58

Isequilla N. L., Benaglia P., Ishwara-Chandra C. H., Intema H., 2020, Boletin de la Asociacion Argentina de Astronomia La Plata Argentina, 61B, 124

Jones T. J., Garwood R., Dickey J. M., 1988, ApJ, 328, 559

Knödlseder J., Cerviño M., Le Duigou J. M., Meynet G., Schaerer D., von Ballmoos P., 2002, A\&A, 390, 945

Laing R. A., Riley J. M., Longair M. S., 1983, MNRAS, 204, 151

Lanzuisi G., et al., 2013, MNRAS, 431, 978

Leahy J. P., Williams A. G., 1984, Monthly Notices of the Royal Astronomical Society, 210, 929

Martí J., Paredes J. M., Ishwara Chandra C. H., Bosch-Ramon V., 2007, A\&A, 472, 557

Masetti N., et al., 2013, A\&A, 556, A120

Morford J. C., et al., 2020, A\&A, 637, A64

Münch L., Morgan W. W., 1953, ApJ, 118, 161

Neugebauer G., et al., 1984, ApJ, 278, L1

Ocran E. F., Taylor A. R., Vaccari M., Ishwara-Chand ra C. H., Prandoni I., 2020, MNRAS, 491, 1127

Osterbrock D. E., 1989, Astrophysics of gaseous nebulae and active galactic nuclei. .

Pal S., Kumari S., 2021, arXiv e-prints, p. arXiv:2103.15199

Pratap P., McIntosh G., 2005, American Journal of Physics, 73, 399

Reddish V. C., Lawrence L. C., Pratt N. M., 1966, Publications of the Royal Observatory of Edinburgh, 5, 111

Reipurth B., Schneider N., 2008, Star Formation and Young Clusters in Cygnus. ., p. 36

Saikia D. J., Thomasson P., Roy S., Pedlar A., Muxlow T. W. B., 2004, MNRAS, 354, 827

Saxena A., et al., 2018, MNRAS, 480, 2733

Schoenmakers A. P., de Bruyn A. G., Röttgering H. J. A., 
van der Laan H., Kaiser C. R., 2000, Monthly Notices of the Royal Astronomical Society, 315, 371

Schulte D. H., 1956a, ApJ, 123, 250

Schulte D. H., 1956b, ApJ, 124, 530

Schulte D. H., 1958, ApJ, 128, 41

Setia Gunawan D. Y. A., de Bruyn A. G., van der Hucht K. A., Williams P. M., 2003, ApJS, 149, 123

Skrutskie M. F., et al., 2006, AJ, 131, 1163

Taylor A. R., Goss W. M., Coleman P. H., van Leeuwen J., Wallace B. J., 1996, ApJS, 107, 239

Werner M. W., et al., 2004, ApJS, 154, 1

Williams P. M., Dougherty S. M., Davis R. J., van der Hucht K. A., Bode M. F., Setia Gunawan D. Y. A., 1997, MNRAS, 289,10

Wright E. L., et al., 2010, AJ, 140, 1868

Zoonematkermani S., Helfand D. J., Becker R. H., White R. L., Perley R. A., 1990, ApJS, 74, 181 